\title{
MOG cosmology without dark matter and the cosmological constant
}

\author{
Zahra Davari ${ }^{1}$ and Sohrab Rahvar ${ }^{1}$ \\ 1 Department of Physics, Sharif University of Technology, P.O.Box 11365-9161, Tehran, Iran
}

Accepted ?, Received ?; in original form August 17, 2021

\begin{abstract}
In this work, we investigate the MOdified Gravity (MOG) theory for dynamics of the universe and compare the results with the $\Lambda \mathrm{CDM}$ cosmology. We study the background cosmological properties of the MOG model and structure formation at the linear perturbation level. We compare the two models with the currently available cosmological data by using statistical Bayesian analyses. After obtaining updated constraints on the free parameters, we use some methods of model selection to assist in choosing the more consistent model such as the reduced chi-squared $\left(\chi_{\mathrm{red}}^{2}\right)$ and a number of the basic information criteria such as the Akaike Information Criterion (AIC), the Bayes factor or Bayesian Information Criterion (BIC), and Deviance Information Criterion (DIC). MOG model appears to be consistent with the $\Lambda$ CDM model by the results of $\chi_{\text {red }}^{2}$ and DIC for an overall statistical analysis using the background data and the linear growth of structure formation.
\end{abstract}

Key words: cosmology: methods: analytical - cosmology: theory - dark energy - modified gravity

\section{INTRODUCTION}

The impressive and wonderful success of the standard $\Lambda \mathrm{CDM}$ model across many different scales and in a wide range of cosmological observations indicate that $\Lambda \mathrm{CDM}$ is superior to other models due to its simplicity and fewer free parameters. However, this standard model involves many observational problems in cosmology, such as the lack of the nature of dark matter particles (DM) as well as the dark energy (DE). Moreover, recent advances in precision cosmology have yielded discrepancies in observations at different redshifts, in particular, the following tensions in the $\Lambda \mathrm{CDM}$ model: $H_{0}$ tension (Aghanim et al. 2018; Riess et al. 2019), $S_{8}$ tension (Kumar et al. 2019), Cosmic Microwave Background (CMB) high-low 1 tension (Hinshaw et al. 2013), Baryon Acoustic Oscillation (BAO) ly- $\alpha$ tension (McDonald et al. 2005; Slosar et al. 2013), the suppress of the high angular scale correlation in CMB power spectrum (Bernui et al. 2018), signature for the violation of the statistical isotropy of CMB (Schwarz et al. 2016), which have opened the door to several alternative models. According to the $\Lambda \mathrm{CDM}$ model, only about $5 \%$ of the universe is either visible or detectable. This fact has become a powerful motivator for the search for alternative explanations that can explain cosmic observations without regard to dark matter or cosmological constant or both of them.

One of these alternative models is the fully covariant and relativistic modified gravity (MOG) theory (Moffat 2006). The MOG theory or Scalar-Tensor-Vector-Gravity (STVG) has been explained successfully the rotation curves of galaxies and account for galaxy cluster masses(Moffat \& Rahvar 2013,
2014; Moffat \& Toth 2015; Brownstein \& Moffat 2006; Green \& Moffat 2019; Davari \& Rahvar 2020), velocity dispersions of satellite galaxies, globular clusters (Moffat 2020), and the Bullet Cluster without resorting to cold dark matter (Israel $\&$ Moffat 2018). It is also shown that MOG theory satisfies the weak equivalence principle. Also this theory is in agreement with gravitational wave event of GRB170817A and corresponding gamma ray burster event of GRB170817A which could be explained as the merge of the neutron stars (Green et al. 2018).

The action of the MOG theory, in addition to EinsteinHilbert and matter terms, includes a massive vector field and three scaler fields corresponding to running values of the gravitational constant, the vector field coupling constant, and the mass of vector field.

In this paper, we investigate the dynamics of the universe using MOG taking into account baryonic matter and radiation as the Energy- Momentum parameters of the universe. Also, we investigate linear structure formation in this model.

This paper is structured as follows: In section (2), we first introduce the MOG theory and then review the key features of the background evolution of the universe in the MOG theory. In section (3), after that, we derive the main equations governing the evolution of baryonic matter in the linear perturbation and investigate the growth of matter perturbations in the MOG model. We introduce the available cosmological data at background and perturbation levels and we perform a numerical Markov Chain Monte Carlo (MCMC) analysis in order to constraint the free parameters of the models and compare them to each other in sections (4) and (5). After that, we deal with the evolution of a number of some main 
cosmological quantities with the best results obtained with the data in the section (6). Finally, our findings are drawn in section (7).

\section{BACKGROUND EVOLUATION IN THE MOG COSMOLOGY}

In order to study and evaluate the MOG theory at the background level, it is necessary to first obtain the main equations governing the evolution of the cosmic background within the MOG theory.

The MOG theory consists of a massive vector field $\phi_{\mu}$, three scalar fields of (i) G, the coupling strength of gravity which can also be considered as a scalar field, (ii) $\omega$, which describes the coupling strength between the vector field and matter and (iii) $\mu$, that related to the mass of the vector field (Moffat 2006; Moffat \& Toth 2009). The MOG action which includes scalar, vector and tensor fields is given by $S=S_{\phi}+S_{G}+S_{S}+S_{M}$, that

$$
\begin{gathered}
S_{G}=-\frac{1}{16 \pi} \int \frac{1}{G}(R+2 \Lambda) \sqrt{-g} d^{4} x \\
S_{\phi}=-\frac{1}{4 \pi} \omega \int\left[\frac{1}{4} B^{\mu \nu} B_{\mu \nu}-\frac{1}{2} \mu^{2} \phi_{\mu} \phi^{\mu}+V_{\phi}(\phi)\right] \sqrt{-g} d^{4} x \\
S_{S}=-\int \frac{1}{G}\left[\frac{1}{2} g^{\alpha \beta}\left(\frac{\nabla_{\alpha} G \nabla_{\beta} G}{G^{2}}+\frac{\nabla_{\alpha} \mu \nabla_{\beta} \mu}{\mu^{2}}\right)+\right. \\
\left.\frac{V_{G}(G)}{G^{2}}+\frac{V_{\mu}(\mu)}{\mu^{2}}\right] \sqrt{-g} d^{4} x
\end{gathered}
$$

where $S_{M}$ is the matter action, $B_{\mu \nu}=\partial_{\mu} \phi_{\nu}-\partial_{\nu} \phi_{\mu}$ is the Faraday tensor for the vector field and $V_{\phi}(\phi), V_{G}(G)$ and $V_{\mu}(\mu)$ represent potentials corresponding to the vector field and the scalar fields (Carolina et al. 2020). The symbol $\nabla_{\nu}$ shows the covariant derivative with respect to the metric $g_{\mu \nu}$ and the symbols $R$ and $g$ are the Ricci-scalar and the determinant of the metric tensor, respectively.

We assume that the universe is isotropic and homogeneous. The observed isotropy of cosmic microwave background radiation is the main evidence that confirms this cosmological principle. We base our cosmology on Friedmann-LemaitreRobertson-Walker (FLRW) metric:

$d s^{2}=d t^{2}-a^{2}(t)\left(\frac{d r^{2}}{1-k r^{2}}+r^{2}\left(d \theta^{2}+\sin ^{2} \theta d \phi^{2}\right)\right)$,

where $a(t)$ is the scale factor and $k=0,-1,+1$ corresponds to flat, open and closed spatial geometry, respectively. Since observations point to almost spatial flatness, we shall restrict ourselves to $k=0$ throughout the work. Due to the symmetries of the FLRW background spacetime, we set $\phi_{i}=0(i=1,2,3)$ and $B_{\mu \nu}=0$ and also in Moffat \& Toth (2009) is supposed the value of $\mathrm{G}$ changes sharply, although smoothly; meanwhile, the scalar fields $\omega$ and $\mu$ remain constant. We use the energy-momentum tensor of a perfect fluid:

$T^{\mu \nu}=(\rho+p) u^{\mu} u^{\nu}-p g^{\mu \nu}$,

where $u^{\mu}=d x^{\mu} / d s$ and $\rho$ and $\mathrm{p}$ are the density and pressure of matter. As it has discussed in section (1) in the MOG theory, we do not include dark matter and constant cosmological as dark energy to explain astrophysical and cosmological observations. So we have

$\rho=\rho_{B}+\rho_{R}+\rho_{G}+\rho_{k}+\rho_{V}$,

where $\rho_{B}$ and $\rho_{R}$ denote the density of baryons and radiation(photons and neutrinos) and $\rho_{G}, \rho_{k}$ and $\rho_{V}$ are the kinetic, the potential of the Brans-Dicke (the scalar field $G$ ) terms, respectively. They are further explained in the following. Since radiation and baryons do not interact with each other, thus they follow the standard equations of evolution i.e $\rho_{R}(a)=\rho_{0 r} a^{-4}$ and $\rho_{B}(a)=\rho_{0 b} a^{-3}$, respectively.

The modified Einstein field equations for the MOG theory can be written in an explained geometric structure of the universe follow as: (Moffat 2014):

$$
\begin{aligned}
& H^{2}+\frac{k}{a^{2}}=\frac{8 \pi G}{3} \rho-\frac{4 \pi}{3}\left(\frac{\dot{G}^{2}}{G^{2}}\right)+\frac{8 \pi}{3}\left(\frac{V_{G}}{G^{2}}\right)+H \frac{\dot{G}}{G}, \\
& \frac{\ddot{a}}{a}=-\frac{4 \pi G}{3}(\rho+3 p)+\frac{8 \pi}{3}\left(\frac{\dot{G}^{2}}{G^{2}}\right)+\frac{8 \pi}{3}\left(\frac{V_{G}}{G^{2}}\right)+H \frac{\dot{G}}{2 G}+\frac{\ddot{G}}{2 G}-\frac{\dot{G}^{2}}{G^{2}},
\end{aligned}
$$

where an overhead denotes derivative with respect to the cosmic time and $H \equiv \dot{a} / a$ is the Hubble parameter. On the other hand, the equation of motion for the scalar field $\mathrm{G}$ obtain as:

$\ddot{G}+3 H \dot{G}-\frac{3}{2} \frac{\dot{G}^{2}}{G}+\frac{3}{G} V_{G}-V_{G}^{\prime}-\frac{3 G}{8 \pi}\left(\frac{\ddot{a}}{a}+H^{2}\right)=0$,

where $V_{G}^{\prime}=d V_{G} / d G$. We can rewrite the Friedmann equations into the familiar form:

$H^{2}=\frac{8 \pi G}{3} \sum_{i} \rho_{i}=\frac{8 \pi G}{3}\left[\rho+\rho_{V}+\rho_{k}+\rho_{G}\right]$,

and

$$
\begin{aligned}
\frac{\ddot{a}}{a} & =-\frac{4 \pi G}{3}\left[\rho(1+3 w)+\rho_{V}\left(1+3 w_{V}\right)+\rho_{k}\left(1+3 w_{k}\right)\right. \\
& \left.+\rho_{G}\left(1+3 w_{G}\right)\right]-\frac{\dot{G}^{2}}{G^{2}}+\frac{\ddot{G}}{2 G},
\end{aligned}
$$

where $\rho_{V}=\frac{1}{G}\left(\frac{V_{G}}{G^{2}}\right), \rho_{k}=-\frac{1}{2 G}\left(\frac{\dot{G} 2}{G^{2}}\right)$ and $\rho_{G}=\frac{3 H \dot{G}}{8 \pi G^{2}}$ are the potential, the kinetic and Brans-Dicke terms, respectively. The equations of state (EoS) associated with $\rho_{k}, \rho_{V}$, and $\rho_{G}$ by comparing the equations (9) and (6) are obtained 1,-1 and $-2 / 3$, respectively (Moffat \& Toth 2007).

It has been shown that for the proper description of cosmological quantities such as evolutionary stages of the universe, the age and the redshift space distortion (RSD) data, $V_{G}$ must be different from 0 , in addition, in previous studies of the MOG theory had considered $V_{G}$ as a constant (Toth 2010; Jamali et al. 2020). In this work, we will consider it as a power-law function inspired by the original Rata \& Peebles (1988) potential: $V_{G}=V_{G 0} G^{-\beta}$ where $V_{G 0}$ and $\beta$ are free parameters. It should be noted that another example of Scalar-Tensor gravity theories is Brans-Dicke theory (BD). The gravitational constant, $\mathrm{G}$ in Brans-Dicke theory assumed as an inverse of the scalar field $\phi$. The field equation in BD theory contains a dimensionless parameter, $\omega_{B D}$, called the BransDicke coupling constant and it can be determined by fitting to the astronomical observations and astrophysical experiments. There are similarities between BD theory and the MOG theory that we have considered but the equations (5) to (7) are 
different from the relations obtained for Brans-Dicke theory in references Hrycyna et al. (2014); Solà Peracaula et al. (2020) and Pérez (2021) since we assume a potential function with a free coefficient, $\beta$ that it is different from similar works in Brans-Dicke theory. Also here, we do not consider both the dark matter and the cosmological constant in the content of the universe.

It is appropriate to represent equations in terms of the scale factor using $\frac{d}{d t}=a H \frac{d}{d a}$ for example we can rewrite the equation (5) as

$$
H(a)=\sqrt{\frac{H_{0}^{2} \tilde{G}(a)\left[\Omega_{b 0} a^{-3}+\Omega_{r 0} a^{-4}\right]+\frac{8 \pi}{3} \tilde{V}_{G 0} \tilde{G}(a)^{-\beta-2}}{1+\frac{4 \pi}{3} a^{2} \frac{\tilde{G}^{\prime 2}(a)}{\tilde{G}^{2}(a)}-a \frac{\tilde{G}^{\prime}(a)}{\tilde{G}(a)}}} ;
$$

the current critical energy density are defined as $\rho_{c 0}=$ $3 H_{0}^{2} / 8 \pi G(1)$ and the present values of the cosmological density parameters $\Omega_{i}=\rho_{i} / \rho_{c 0}$, we also define $\tilde{G}(a)=$ $G(a) / G(1)$ and $\tilde{V}_{G 0}=V_{G 0} G(1)^{-\beta-1}$. In order to find the dynamics of $\mathrm{G}(\mathrm{a})$, we solve numerically in the equation (7) with suitable initial conditions. In most previous literature, the initial conditions were considered as follows in the present time $(a=1): G(1)=G_{N}$ and $G^{\prime}(1)=0$. In this work, we take on an ansatz of the power-law to determine the initial conditions in the early universe:

$a=a_{0} t^{n_{1}}, \quad \tilde{G}=G_{0} t^{n_{2}} ;$

where $\mathrm{t}$ is normalized to $t_{0}$ (the age of the universe) in the present time then for matter dominated epoch we have zero pressure $(P=0)$ and $\rho \propto a^{-3} \propto t^{-3 n_{1}}$. Substituting these relations in the field equation leads to

$n_{1}=\frac{2(\beta+3)}{3(\beta+2)}, \quad n_{2}=\frac{2}{\beta+2} ;$

and

$G_{0}=\left[\frac{12 \pi \tilde{V}_{G_{0}}(\beta+2)^{2}}{(6-24 \pi+\beta)}\right]^{\frac{1}{\beta+2}}$,

where we can constrain the parameters of model with the numeric value of $G_{0}$. For radiation dominated epoch, we obtain $n_{1}=\frac{(\beta+3)}{2(\beta+2)}$. In the GR Einstein- de Sitter universe model (EdS), for matter or radiation, we have $a \propto t^{2 / 3}$ or $a \propto t^{1 / 2}$ but here we obtain $a \propto t^{2(\beta+3) / 3(\beta+2)}$ or $a \propto t^{2(\beta+3) / 4(\beta+2)}$ for matter and radiation dominated epoch, respectively where the MOG model behavior similar to EdS model for $\beta \rightarrow \infty$. We can substitute the cosmic time in (11) in terms of the scale factor. We use $H=n_{1} / t$ and can obtain $t^{2}=3 n_{1}^{2} / 8 \pi G_{0} \rho_{B}$ for the matter dominated epoch where $\rho_{B}=\rho_{c 0} \Omega_{b 0} a^{-3}$. On the other hand, with an effective gravitational constant of $G_{\text {eff }} \approx 6 G_{N}$, the effective baryonic matter taking into account the factor of six and contribute $30 \%$ of the critical density of the universe, therefore no nonbaryonic dark matter is needed to explain a deficiency in matter density. We use $t^{2}=n_{1}^{2} a^{3} / 6 H_{0}^{2} \Omega_{b 0}$ and determine $\tilde{G}$ as a function of the scale factor as

$\tilde{G}(a)=\left(\frac{8 \pi \tilde{V}_{G_{0}}(\beta+3)^{2}}{9 \times 10^{4} h^{2} \Omega_{b 0}(6-24 \pi+\beta)}\right)^{\frac{1}{\beta+2}} a^{\frac{3}{\beta+3}}$,

where $\mathrm{h}$, the reduced Hubble constant is usually defined according to $H_{0} \equiv 100 h$. As $\tilde{V}_{G_{0}}>0$, the equation (14) imposes
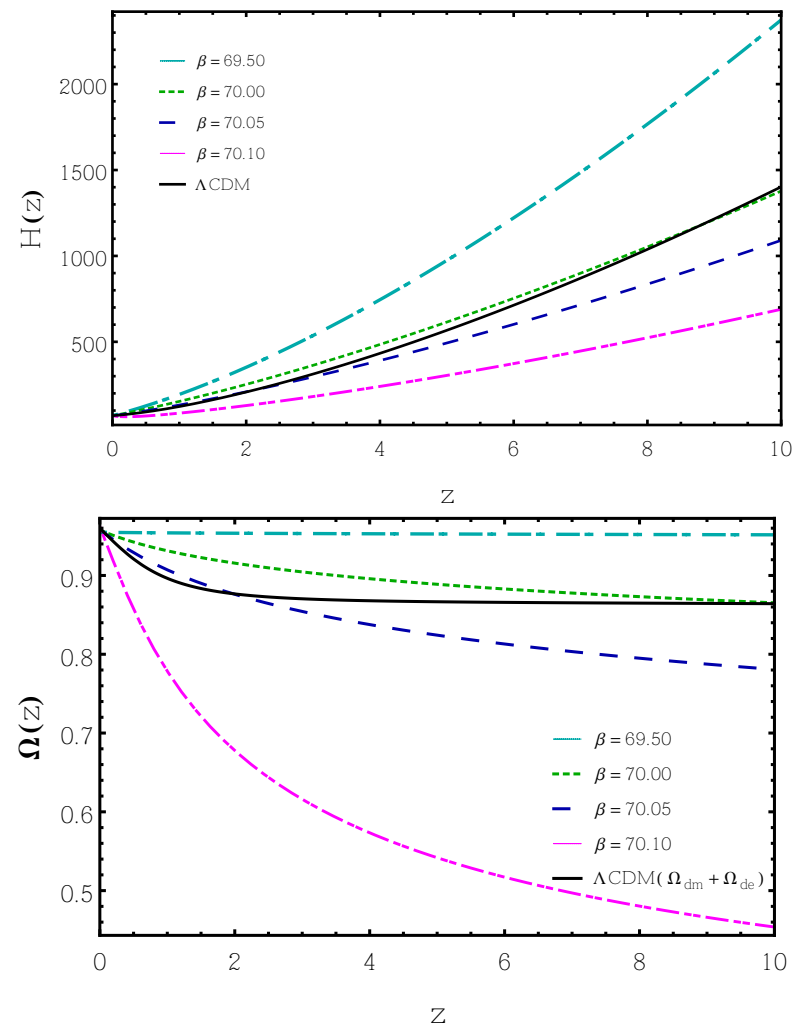

Figure 1. The redshift evolution of $H(z)$ (top panel), $\Omega(z)$ (bottom panel). The long dashed, dotted, dashed and dot-dashed curves correspond to MOG models with $\beta=69.5,70.0,70.05$ and 70.10 , respectively. We show the concordance $\Lambda$ CDM model with the black solid curve. .

$\beta>24 \pi-6 \simeq 69.4$. In the following, we survey some of the most important parameters describing the expansion of the universe such as the present value of the Hubble parameter known as Hubble constant and the fraction $\Omega=\rho / \rho_{c}$, where $\rho_{c}$ is the critical density that corresponds to a flat universe. We survey the behavior of the Hubble parameter in MOG cosmology since the Hubble expansion affects the growth of matter perturbations. In Figure (1), we show the redshift evolution of the Hubble parameter, $H(z)$ (top panel) for different values of the MOG parameter $\beta=69.50$ (long dashed cyan line), $\beta=70$ (dotted green line), $\beta=70.05$ (dashed blue line) and $\beta=70.10$ (dot-dashed pink line). We also plotted the concordance $\Lambda \mathrm{CDM}$ model for comparison( the solid black line). As expected, it depends on the choice of $\beta$ and we see that in the case of $\beta<70$, we have $H_{M O G}(z)>H_{\Lambda}(z)$, while the opposite holds for $\beta>70$ and here the MOG has the best compatibility for $\beta=70.05$ with observational data and for $\beta=70$ has a similar behavior of the standard model. In bottom panel of Figure (1), we plot the evolution of the energy density parameter of the scalar field, $\Omega_{V}(z)$ for all aforementioned values of $\beta$. As mentioned it played both dark matter and dark energy role. The panel shows $\Omega_{V}(z)$ for $\beta=70$ has similar behavior to $\Omega(z)$ in the $\Lambda \mathrm{CDM}$ case in large value of redshift. It should be noted that in these two panels of Figure (1) we adopted for MOG models $\left(\Omega_{b 0}=0.04, H_{0}=70, V_{G 0}=0.70\right)$ and also for the $\Lambda \mathrm{CDM}$ $\left(\Omega_{b 0}=0.04, \Omega_{d m 0}=0.26, H_{0}=70\right)$. 
To further investigate the properties of the MOG theory, we define two additional parameters: the effective equation of state parameter, $w_{\text {eff }}$ and the deceleration parameter, $\mathrm{q}$. $w_{\text {eff }}$ is defined to include all components of the energy budget of the cosmos into an effective density $\rho_{\text {eff }}$ and effective pressure $p_{\text {eff }}=w_{\text {eff }} \rho_{\text {eff }}$, such that the Friedmann equations for $\Lambda \mathrm{CDM}$ model is written as $H^{2}=8 \pi G / 3 \rho_{\text {eff }}$ and $\ddot{a} / a=-4 \pi G / 3\left(1+3 w_{\text {eff }}\right) \rho_{\text {eff }}$. It is given namely: $w_{\text {eff }}=$ $\frac{p_{\text {tot }}}{\rho_{\text {tot }}}=-1-\frac{2 \dot{H}}{3 H^{2}}$. Using the equations (8) and (6), we can write for MOG theory:

$w_{\text {eff }}=-1-\frac{2 \dot{H}}{3 H^{2}}-\frac{2\left(-\ddot{G} / 2 G+\dot{G}^{2} / G^{2}\right)}{3 H^{2}}$.

In the top panel of Figure (2), we plot the evolution of the $w_{\text {eff }}$ as a function of $\mathrm{z}$. As expected, at the time of the current accelerated expansion $w_{\text {eff }}$ at present time obtain $-0.02,-0.31,-0.57$ and -1.85 for $\beta=69.5,70.0,70.05$ and 70.10 , respectively and for $\Lambda \mathrm{CDM}$ is -0.70 . We observe that for $\beta>70.08$, we reach the phantom regime prior to the present time $(w<-1)$. In the case of $\beta=70.10$ the EoS parameter crosses the phantom line $w=-1$ at the epoch of $z \sim 0.2$, while for $\beta<70.10$ it remains in quintessence regime for all $\mathrm{z}$.

If we compare this panel with $\mathrm{H}(\mathrm{z})$ parameter in Figure (1) we observe for the quintessence MOG models $(\beta<70.08)$, $H_{M O G}(z)>H_{\Lambda}$ for all redshifts which means that the corresponding cosmic expansion is larger than that of the concordance $\Lambda$ CDM model. Finally as a complementary information, in the bottom panel of the Figure (2), we present the evolution of a deceleration parameter $q=-\frac{\ddot{a}}{a H^{2}}=-1-\frac{\dot{H}}{H^{2}}$ in term of the redshift. Recall that $\mathrm{q}$ for determining the accelerated phase of the expansion $(q<0)$ or decelerated phase $(q$ $>0$ ) can be used and $q=0$ indicates the position of the transition point from decelerated expansion to accelerated expansion in the universe. As expected, at the time of the current accelerated expansion, $\mathrm{q}$ is negative but we see in this panel only for two cases of MOG models that $\beta>70$, q are negative. The transition redshift from deceleration to acceleration for $\beta=70.05$ is at $z_{t}=0.68$, which is in good agreement with the values obtained for the $\Lambda \mathrm{CDM}\left(z_{t}=0.67\right)$. Also q tends to $\frac{1}{2}$ that representing the deceleration phase in the matter dominated epoch. From the top panel of Figure (2), we can see for MOG model that for large redshifts $w_{\text {eff }}$ approach into small negative value but not zero, unlike to $\Lambda$ CDM model, however from the bottom panel of Figure (2) we have deceleration phase for the large $\mathrm{z}$ (in the early universe). Figure (2) shows the fact that the effective equation of state and the deceleration parameter for both models are consistent and behave in a similar way. These results are in good settlement with the values obtained in (Farooq et al. 2017).

We show in Figure (3) G increase with scale factor for all values of free parameter $\beta$. In the following, we will present an argument based on simplified analytical calculations that MOG theory results in the accelerated expansion of the early universe similar to the standard period of cosmic inflation. It is obtained by substituting the relations (11) in the second Friedmann equation (6):

$\frac{\ddot{a}}{a}=-\frac{6+\beta-48 \pi}{9(\beta+2)^{2}}$,

which is positive given the condition we have already obtained for the value $\beta>24 \pi-6$.
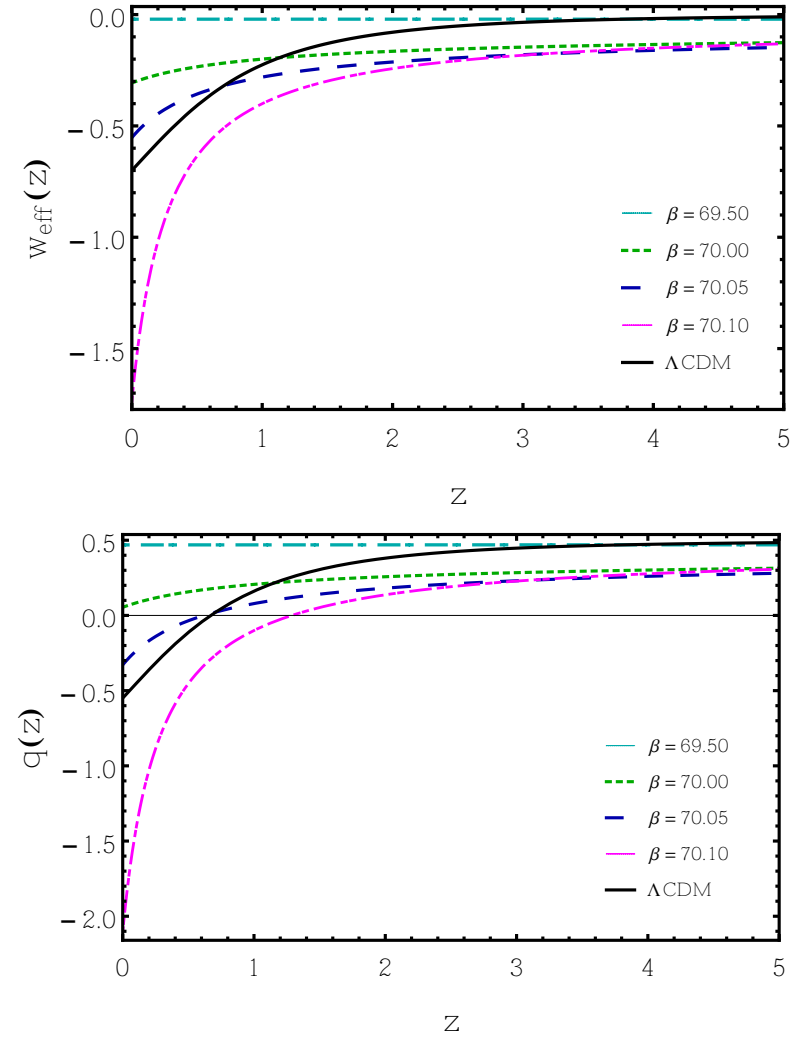

Figure 2. The redshift evolution of the deceleration parameter $\mathrm{q}(\mathrm{z})$ (top panel), the effective Equation of State, $\mathrm{w}_{\text {eff }}$ (bottom panel). The color of the curves used is similar to the Figure (1).

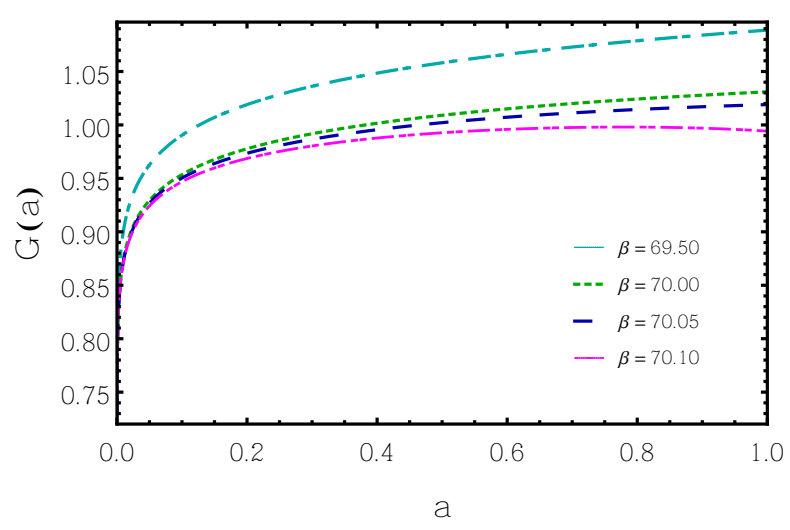

Figure 3. The evolution of the effective gravitational constant. The values of the parameters used are similar to the Figure (1).

\section{PERTURBATION IN MOG COSMOLOGY}

A fundamental issue in cosmology is the formation of cosmic structures so, in order to efficiently distinguish between MOG and $\Lambda \mathrm{CDM}$ models, the evolution of matter density perturbations must be compared with cosmological observations. In the following given that in Section (2) we considered the FRLW metric for determining dynamics of universe at the background level and also supposed that $G$ is only dynamical scale field therefore we will consider the evaluation of perturbations in the linear regime $(\delta \ll 1)$. However, we re- 
mind other fields $(\mu$ and $\omega$ ) must be considered to study the nonlinear growth rate of $\operatorname{structures}(\delta>1)$. Since, we will survey the linear perturbations of the metric tensor and the matter and scalar field perturbations.

The Jeans equation in the context of GR gives the density perturbation equation for the non-relativistic singlecomponent fluid as follow:

$$
\ddot{\delta}+2 H \dot{\delta}+\left(\frac{c_{s}^{2} a_{0}^{2} k^{2}}{a^{2}}-4 \pi G(a) \rho\right) \delta=0,
$$

where $c_{s}=\sqrt{d p / d \rho}$ is the speed of sound and the quantity $k / a$ is called the comoving wave number and we assumed G(a) in the context of MOG (Moffat \& Toth 2007).

The factor sign in parentheses determines the nature of the solutions of Equation (17). When the pressure is larger than the gravity term (in other words for large k) we have oscillatory solution and on the other hand when gravity term predominates (for small k) the density contrast is growing. When gravity and the pressure terms are equal, the Jeans wave number is given by:

$k_{J}=\frac{a}{a_{0}} \frac{\sqrt{4 \pi G(a) \rho}}{c_{s}} ;$

corresponding to the Jeans length $\lambda_{J}=2 \pi / k_{J}$ (wavelength) and for non-relativistic matter $k_{J} \gg\left(a / a_{0}\right) H$ and $c_{s} \ll 1$.

$V_{G}$ s do not couple to photons since they are electrically neutral and they can be considered as almost pressure-less, so the pressure gradient term in the Jeans equation is absent and $c_{s} \sim 0$. The wavelength is approximately zero and we obtain

$$
\ddot{\delta}+2 H \dot{\delta}-4 \pi G(a) \rho \delta=0 .
$$

It is more appropriate to write equation (19) in terms of the scale factor as:

$\delta^{\prime \prime}(a)+\left(\frac{3}{a}+\frac{H^{\prime}(a)}{H(a)}\right) \delta^{\prime}(a)-\frac{3}{2} \frac{\Omega_{b 0} G(a)}{a^{5} H^{2}(a) / H_{0}^{2}} \delta(a)=0$,

which is solved numerically taking an initial contrast of $\delta_{m i}=5 \times 10^{-5}$ at an initial scale factor of $a_{i}=10^{-4}$. This means that we study the evolution of structure in a matter dominated era and considering $G_{\text {eff }} \approx 6 G_{N}$.

In order to compare these models with observational data in perturbation level, we calculate the so-called linear growth rate, namely the logarithmic derivative of the linear density contrast with respect to the scale factor:

$f(a)=\frac{d \ln \delta_{m}}{d \ln a}$.

The growth rate has been observed in the redshift ranging of $z \in[0.02,1.4]$ by several surveys. In the top panel of Figure (4), we plot the linear growth factor $f(z)$ for the different values of $\beta$. We see in this panel that the amplitude of matter perturbations at low redshifts decreases due to the accelerated expansion of recent times in the universe. This panel shows that the growth of perturbations is negligible at high redshifts and consequently, the growth function goes to unity value, which corresponds to the matter dominated universe but it did not happen for value $\beta>70.08$. We observe that the suppression of the amplitude of matter fluctuations in the MOG cosmologies starts sooner as compared with the concordance $\Lambda \mathrm{CDM}$ model. Another important quantity in the perturbation level to consider is the redshift-dependent
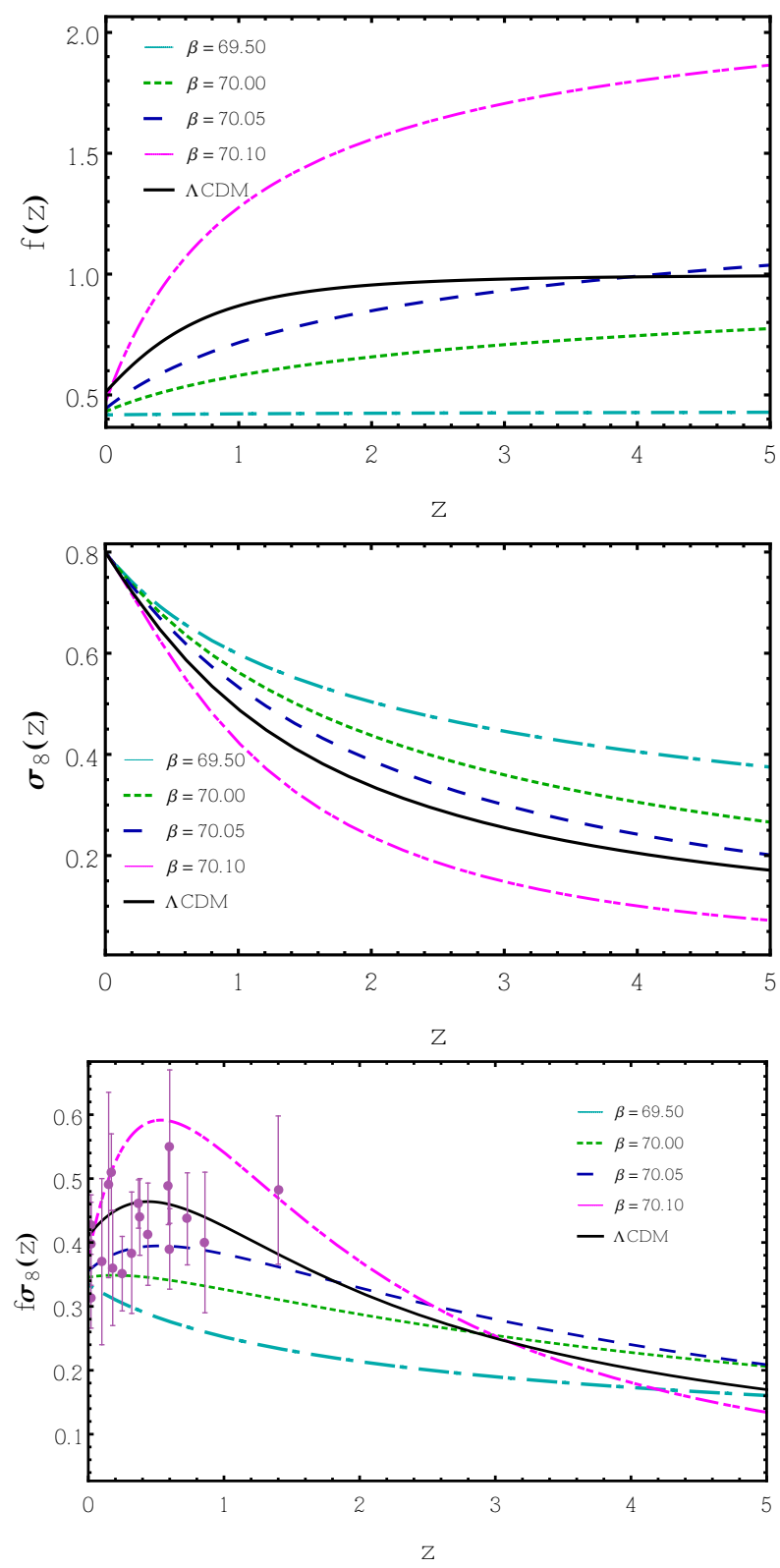

Figure 4. Redshift evolution of matter growth rate function $f(z)$ (top panel), variance of perturbations $\sigma_{8}(z)$ (middle panel) in the context and (bottom panel) the long term evolution of $f(z) \sigma_{8}(z)$ compared to observational growth rate data points in MOG cosmology(Nesseris et al. 2017). Here, we show the concordance $\Lambda$ CDM model by solid black curve.

rms fluctuations of the linear density field within spheres of radius $R=8 h^{-1} \mathrm{Mpc}, \sigma_{8}(z)$ and it is computed as follows

$\sigma_{8}(a)=\sigma_{8}(a=1) \frac{\delta_{m}(a)}{\delta_{m}(a=1)}$.

We see the evolution of $\sigma_{8}(z)$ as a function of redshift $z$ in the middle panel of Figure (4). It shows that opposite to the behavior of growth rate function, $\sigma_{8}(z)$ in MOG models for $\beta<70.08$ is larger than the one in the case of the $\Lambda \mathrm{CDM}$ cosmology. A robust measurable quantity in perturbation surveys is $f(z) \sigma_{8}(z)=f \sigma_{8}(z)$. This quantity is shown in the bottom panel of Figure (4). As expected, the quantity 
Table 1. Summary of the flat priors on the cosmological parameters assumed in this work.

\begin{tabular}{cc}
\hline Parameter & Prior range \\
\hline \hline$\Omega_{b 0}$ & {$[0.01,0.2]$} \\
$\Omega_{d m 0}$ & {$[0.01,0.5]$} \\
$H_{0}$ & {$[50,100]$} \\
$\sigma_{80}$ & {$[0.5,1]$} \\
$r_{\mathrm{d}, \mathrm{fid}}$ & $>0$ \\
$\beta$ & $>69.5$ \\
$V_{G 0}$ & $>0$ \\
\hline
\end{tabular}

$f(z) \sigma_{8}(z)$ is sensitive to the value of the parameters $\beta$. For simplicity, we will denote $\sigma_{8}(z=0)=\sigma_{80}$ when there is no ambiguity. It should be noted that in all panels of Figure (4) we adopted in addition the initial value of the parameters similar to Figure (1), $\sigma_{80}=0.80$ for all models.

\section{MOG COSMOLOGY VERSUS DATA}

In what follows, our aim is to constrain the parameters of the MOG and $\Lambda \mathrm{CDM}$ models using the currently available cosmological data at the background and perturbation levels via the MCMC method. We use the maximum likelihood estimation as the fitting method. In this method according to $\mathcal{L}_{\text {tot }} \simeq e^{-\chi_{\text {tot }}^{2} / 2}$, the total likelihood function is maximized by minimizing $\chi_{\text {tot }}^{2}$. To determine $\chi_{\text {tot }}^{2}$ we use the following observational data set: big bang nucleosynthesis (BBN, 1 point), the Hubble evolution data ( $\mathrm{H}(\mathrm{z}), 31$ points), type Ia supernova data (SNIa, 40 points from Pantheon binned sample), the cosmic microwave background radiation data (CMB, 2 points) and the baryon acoustic oscillation data (BAO, 14 points). Therefore $\chi_{\text {tot }}^{2}$ for the first analysis is given by the relation

$\chi_{\mathrm{tot}, 1}^{2}(\mathbf{p})=\chi_{\mathrm{BBN}}^{2}(\mathbf{p})+\chi_{\mathrm{OH} D}^{2}(\mathbf{p})+\chi_{\mathrm{SN}}^{2}(\mathbf{p})+\chi_{\mathrm{CMB}}^{2}(\mathbf{p})+\chi_{\mathrm{BAO}}^{2}(\mathbf{p})$,

where the statistical vector $\mathbf{p}$ in this analysis is $\left(\Omega_{b 0}, \Omega_{d m 0}, H_{0}, r_{\mathrm{d}, \text { fid }}\right), \quad\left(\Omega_{b 0}, H_{0}, r_{\mathrm{d}, \mathrm{fid}}, V_{\mathrm{G} 0}, \beta\right)$ for $\Lambda \mathrm{CDM}$, MOG models, respectively. We also include perturbation observables in our second analysis, specifically we use 18 independent data of the growth rate that were obtained from RSDs of various galaxy surveys so $\chi_{\text {tot }, 2}^{2}\left(\mathbf{p}^{\prime}\right)=\chi_{\text {tot, } 1}^{2}(\mathbf{p})+$ $\chi_{\mathrm{fs}}^{2}\left(\mathbf{p}^{\prime}\right)$. In second analysis, the statistical vector $\mathbf{p}^{\prime}$ also includes $\sigma_{80}=\sigma_{8}(z=0)$ in addition to the parameters mentioned above for $\mathbf{p}$ (Davari et al. 2020). We gauge the statistical strength of RSD data to comparing the result of two analyses. In the Table (1), we listed the prior ranges of free parameters. In following we discuss each $\chi^{2}$ in detail.

\subsection{Big Bang Nucleosynthesis}

One of the most common used primordial elements for constraining the baryon density is the deuterium abundance and the radiative capture of protons in deuterium to produce helium-3 (Nunes \& Bernui 2020). The experimental value for the reaction rate is calculated in Serra et al. (2009), constraining the baryon density to $\Omega_{b 0} h^{2}=0.022 \pm 0.002$. We adopt
Table 2. The best values of the two common parameters obtained with a combination of the binned Pantheon SNIa dataset (40 points) and BBN data.

\begin{tabular}{cccc}
\hline Model & $\Omega_{b 0}$ & $H_{0}$ & $\chi^{2}$ \\
\hline MOG & $0.044_{-0.001}^{+0.001}$ & $70.34_{-1.6}^{+1.6}$ & 67.75 \\
\hline$\Lambda \mathrm{CDM}$ & $0.040_{-0.006}^{+0.003}$ & $74.35_{-6.5}^{+6.5}$ & 67.24 \\
\hline
\end{tabular}

this value as the Gaussian prior likelihood in our analysis. The $\chi_{\mathrm{BBN}}^{2}$ is given by

$\chi_{\mathrm{BBN}}^{2}(\mathbf{p})=\frac{\left[\Omega_{b 0} h^{2}-0.022\right]^{2}}{0.002^{2}}$.

\subsection{Type Ia Supernovae}

The observations of type Ia supernovae (SNIa) is an important probe to probe the expansion history of the universe. This observations cover the scales up to $z \leq 2$. Using the the light-curve templates of SNIa, we can obtain the absolute magnitude of SNIa from the light curves. Currently, there are different collected compilations of SNIa as the Union 2.1 sample by Suzuki et al. (2012) consisting of 580 SNIa data within the range of $[0.015,1.41]$, and full Joint Light-curve Analysis (JLA) sample which contains 740 SNIa within the range of $[0.01,1.2]$ Betoule et al. (2014). The largest combined sample of SNIa which consist of a total of 1048 SNIa in the redshift range $[0.01,2.3]$ presented by Scolnic et al. (2018), is called the Pantheon Sample. In this analyze, we use the binned Pantheon SNIa dataset, which contains 40 data points in range $0.014<z<1.61$. We marginalize over the nuisance parameter analytically in the distance estimates M. In Camarena \& Marra (2018), $\chi_{\mathrm{SN}}^{2}$ was introduced as:

$\chi_{\mathrm{SN}}^{2}(\mathbf{p})=S_{2}(\mathbf{p})-\frac{S_{1}^{2}(\mathbf{p})}{S_{0}}+\ln \frac{S_{0}}{2 \pi}+\ln \left|2 \pi S_{S N}\right|$,

here

$$
\begin{aligned}
& S_{0}=V \cdot S_{S N}^{-1} \cdot V^{T}, \\
& S_{1}=W \cdot S_{S N}^{-1} \cdot V^{T} \\
& S_{2}=W \cdot S_{S N}^{-1} \cdot W^{T},
\end{aligned}
$$

where covariance matrix, $\mathrm{S}$ is from the binned Pantheon sample (considering both systematic and statistical errors) and $\mathrm{V}$ is a row vector of unitary elements and $W_{i}=\mu_{\mathrm{b}, \mathrm{i}}-\mu\left(z_{i}\right)$. The distance modulus, $\mu$, whose theoretical prediction is obtained in term the luminosity distance $d_{L}$ via:

$\mu(z)=5 \log _{10} \frac{d_{L}(z)}{10 \mathrm{pc}}$.

In this way for this set of data, the cosmology-independent normalization constants can be dropped.

Table (2) represent the values of the two common parameters obtained with the help of the sum of SNIa and BBN data. As we observe from Table (2), the SNIa data allow a very large value of the Hubble constant at present and it is actually very close to its local measurements $\left(H_{0}=74.03 \pm 1.42\right)$.

\subsection{Hubble Observational Data}

The Observational Hubble Data (OHD) is a conventional test to check cosmological models which gives a direct measure- 
Table 3. The best values of the two common parameters obtained by using $\mathrm{OHD}(31$ points) and BBN data.

\begin{tabular}{cccc}
\hline Model & $\Omega_{b 0}$ & $H_{0}$ & $\chi^{2}$ \\
\hline MOG & $0.048_{-0.001}^{+0.001}$ & $68.06_{-1.6}^{+0.83}$ & 15.52 \\
\hline$\Lambda \mathrm{CDM}$ & $0.048_{-0.01}^{+0.01}$ & $68.15_{-2.1}^{+2.9}$ & 14.50 \\
\hline
\end{tabular}

ment of the history of the expansion of the universe. The OHD sample is obtained from the differential age of galaxies technique (DAG), and measurements of peaks of BAO. The $\mathrm{H}(\mathrm{z})$ is determined with this method from the following equation:

$H(z)=\frac{-1}{1+z} \frac{d z}{d t}$.

It is worth noting that data points derived from the cosmic chronometers approach, i.e. the massive and passively evolving galaxies (Jimenez \& Loeb 2002) can be used as DAG technique which is cosmological-model-independent. This method is based on the differential age evolution of old elliptical passive-evolving galaxies that are separated by a small redshift interval but formed at the same time. These galaxies have not had any star formation ever since as they have been formed in the early universe, at high redshift, with large stellar mass $>10^{11} \mathcal{M} \odot$. In this way, by calculating the age difference of these galaxies, the derivative $d z / d t$ can be measured from the ratio $\Delta z / \Delta t . \Delta z$ obtained with high accuracy and straightforward from spectroscopic observations. The determination of $\Delta t$ is much more challenging, and this required standard and readable clocks that exist all over the universe so this was the main idea of a cosmic chronometer that stellar evolution could provide such standard clocks. If we found an extremely massive, old, and passively evolving stellar population across a range of redshifts since its stars had formed all simultaneously and evolved passively therefore that would be appropriate to consider as the cosmic chronometer (Moresco et al. 2018).

In this analysis, we use the 31 data points from the recent accurate estimates of $H(z)$ in the redshift range $0.07 \leq z \leq$ 1.965. These data are uncorrelated with the BAO data points and was presented in Marra \& Sapone (2018).

In this case we can write:

$\chi_{O H D}^{2}(\mathbf{p})=\sum_{i} \frac{\left[H\left(z_{i}, \mathbf{p}\right)-H_{i}\right]^{2}}{\sigma_{i}^{2}}$.

where $\sigma_{i}$ is the Gaussian error on the measured value of $H_{i}$. In the Tables (3), we reported the results of our statistical analysis for two models by using OHD and BBN data. As we see two models are consistent with observational data.

\subsection{Cosmic Microwave Background}

The CMB is one of the most significant observables in cosmology. The advantage of CMB data is that it has wellunderstood linear physics and precision to determine the cosmological parameters. Here, we will consider the compressed CMB likelihood (Planck 2018 TT, TE, EE + lowE) from Chen et al. (2019, Table I) on the angular scale of the sound horizon at the last scattering $l_{a}$ and the baryon density $\Omega_{b} h^{2}$. We do not use the spectral index since it does not appear
Table 4. The best values of the two common parameters obtained by using $\mathrm{CMB}$ and BBN data.

\begin{tabular}{cccc}
\hline Model & $\Omega_{b 0}$ & $H_{0}$ & $\chi^{2}$ \\
\hline MOG & $0.048_{-0.001}^{+0.001}$ & $68.51_{-0.52}^{+0.42}$ & 0.04 \\
\hline$\Lambda \mathrm{CDM}$ & $0.044_{-0.002}^{+0.001}$ & $70.01_{-1.5}^{+1.8}$ & 0.03 \\
\hline
\end{tabular}

directly in our calculation and also do not use the shift parameter, $\mathrm{R}$ since it depended on dark matter density. $l_{a}$ is given by:

$l_{a}=\left(1+z_{\star}\right) \pi \frac{D_{A}\left(z_{\star}\right)}{r_{s}\left(z_{\star}\right)}$,

where $r_{s}(z)$ is the comoving sound horizon distance at the recombination is given by $r_{s}(z)=\int_{z}^{\infty} c_{s}(z) / H(z)$ that the sound speed, $c_{s}(z)=\left[3\left(1+3 \frac{\Omega_{b 0}}{4 \Omega_{\gamma 0}(1+z)}\right)\right]^{-1 / 2}$. We set $\Omega_{\gamma 0}=$ $2.469 \times 10^{-5} h^{-2}$ (Hinshaw et al. 2013). The recombination redshift $z_{\star}$ is obtained using the fitting function proposed by $\mathrm{Hu} \&$ Sugiyama (1996) as:

$z_{\star}=1048\left[1+0.00127\left(\Omega_{b 0} h^{2}\right)^{-0.738}\right]\left[1+g_{1}\left(\Omega_{d m 0} h^{2}\right)^{g_{2}}\right]$,

where $g_{1}=\left(0.0783\left(\Omega_{b 0} h^{2}\right)^{-0.238}\right) /\left(1+39.5\left(\Omega_{b 0} h^{2}\right)^{0.763}\right)$ and $g_{2}=(0560) /\left(1+21.1\left(\Omega_{b 0} h^{2}\right)^{1.81}\right)$. It should be noted that for the MOG model, since there is no dark matter, only the first term in the equation (30) is considered. In Aghanim et al. (2020) reported its value $z_{\star}=1089.80 \pm 0.21$ on Planck $\mathrm{TT}, \mathrm{TE}, \mathrm{EE}+$ lowE + lensing. Then we can define $\chi_{C M B}^{2}$ as

$\chi_{\mathrm{CMB}}^{2}(\mathbf{p})=X_{\mathrm{CMB}}^{T} \cdot C_{\mathrm{CMB}}^{-1} \cdot X_{\mathrm{CMB}}$.

For MOG model, we used a prior on $\left(l_{a}, \Omega_{b_{0}} h^{2}\right)$ for the $w$ CDM model derived from the Planck 2018 results (Chen et al. 2019):

$X_{\mathrm{CMB}}=\left(\begin{array}{l}l_{a}-301.462 \\ \Omega_{b_{0}} h^{2}-0.02239\end{array}\right)$,

and

$C_{\mathrm{CMB}}^{-1}=\left(\begin{array}{cc}139.594 & 21357.9 \\ 21357.9 & 28267800\end{array}\right)$.

and also these value for $\Lambda C D M$ be considered as

$X_{\mathrm{CMB}}=\left(\begin{array}{l}l_{a}-301.471 \\ \Omega_{b_{0}} h^{2}-0.02236\end{array}\right)$,

and

$C_{\mathrm{CMB}}^{-1}=\left(\begin{array}{cc}141.675 & 27740 \\ 27740 & 49875900\end{array}\right)$.

We show the values of the two common parameters for two models obtained by using $\mathrm{CMB}$ and $\mathrm{BBN}$ data in the Table (4). A noteworthy point in these results is the large difference between the values of $H_{0}$ obtained for the two models.

\subsection{Baryon acoustic oscillations}

The equilibrium between the pressure and gravity in the baryonic matter of cosmic fluid results in periodic fluctuations of the density which is so called the Baryonic Acoustic 
Table 5. The table shows the distance constraints of BAO measurements for various observational probes. The redshift, the mean value, standard deviation, and the corresponding reference of the observable in each case are reported in the table, respectively.

\begin{tabular}{cccc}
\hline$z_{\text {eff }}$ & Value & Observable & Reference \\
\hline \hline 0.15 & $(664 \pm 25.0)\left(r_{d} / r_{\mathrm{d}, \text { fid }}\right) M p c$ & $D_{V}$ & Ross et al. (2015) \\
0.44 & $(1716 \pm 83.0)\left(r_{d} / r_{\mathrm{d}, \mathrm{fid}}\right) M p c$ & $D_{V}$ & Kazin et al. (2014) \\
0.6 & $(2221 \pm 101.0)\left(r_{d} / r_{\mathrm{d}, \mathrm{fid}}\right) M p c$ & $D_{V}$ & Kazin et al. (2014) \\
1.52 & $(3843 \pm 147.0)\left(r_{d} / r_{\mathrm{d}, \mathrm{fid}}\right) M p c$ & $D_{V}$ & Ata et al. (2018) \\
\hline 0.81 & $(1649.5 \pm 66.0)\left(r_{d} / r_{\mathrm{d}, \mathrm{fid}}\right) M p c$ & $D_{A}$ & Abbott et al. (2019) \\
\hline 0.38 & $(1512 \pm 33.0)\left(r_{d} / r_{\mathrm{d}, \mathrm{fid}}\right) M p c$ & $D_{M}$ & Alam et al. (2020) \\
0.51 & $(1975 \pm 71.0)\left(r_{d} / r_{\mathrm{d}, \mathrm{fid}}\right) M p c$ & $D_{M}$ & Alam et al. (2020) \\
0.61 & $(2307 \pm 80.0)\left(r_{d} / r_{\mathrm{d}, \mathrm{fid}}\right) M p c$ & $D_{M}$ & Alam et al. (2020) \\
2.3 & $(5566 \pm 317.2)\left(r_{d} / r_{\mathrm{d}, \mathrm{fid}}\right) M p c$ & $D_{M}$ & Bautista et al. (2017) \\
2.4 & $(5259.7 \pm 250.5)\left(r_{d} / r_{\mathrm{d}, \mathrm{fid}}\right) M p c$ & $D_{M}$ & Riess et al. (2018) \\
\hline 2.3 & $(1336 \pm 45.7)\left(r_{d} / r_{\mathrm{d}, \mathrm{fid}}\right) M p c$ & $D_{H}$ & Bautista et al. (2017) \\
2.4 & $(1327.4 \pm 53.0)\left(r_{d} / r_{\mathrm{d}, \mathrm{fid}}\right) M p c$ & $D_{H}$ & Riess et al. (2018) \\
\hline 0.38 & $81.2 \pm 3.2 \mathrm{kms}^{-1} \mathrm{Mpc}-1$ & $H$ & Alam et al. (2020) \\
0.51 & $90.9 \pm 3.3 \mathrm{kms}^{-1} \mathrm{Mpc}-1$ & $H$ & Alam et al. (2020) \\
\hline
\end{tabular}

Oscillation (BAO). BAO provides a standard ruler to measure cosmological distances. The BAO signal directly constrains the Hubble parameter $\mathrm{H}(\mathrm{z})$ along the line of sight and also BAO constrains the angular diameter distance $D_{A}(z)=$ $\frac{1}{1+z} \int_{0}^{z} d z^{\prime} / H\left(z^{\prime}\right)$ in a redshift shell. The Dark Energy Survey in first data release provides a measurement of $D_{A}$ at $z_{\text {eff }}=0.81$, using the projected two point correlation function of a sample of over 1.3 million galaxies with measured photometric redshifts, distributed over a footprint of 1336 $\operatorname{deg}^{2}$ (Abbott et al. 2019).

$\mathrm{BAO}$ is determined in the $2 \mathrm{D}$ correlation function while $H(z)$ is determined in $3 \mathrm{D}$ space. In some case it is impossible to combine both quantities can be measured as

$D_{V}(z)=\left[(1+z)^{2} D_{\mathrm{A}}^{2}(z) \frac{z}{H(z)}\right]^{\frac{1}{3}}$.

We present in the Table (5), the isotropic angular diameter distance $D_{V}$ for some $z_{\text {eff }}$ from surveys (Ross et al. 2015; Kazin et al. 2014).

The final galaxy clustering data release of the Baryon Oscillation Spectroscopic Survey (Alam et al. 2020), provides measurements of the comoving angular diameter distance $D_{M}=(1+z) D_{A}(z)$ and Hubble parameter $\mathrm{H}$ at effective redshifts $0.38,0.51$, and 0.61 from the BAO method after applying a reconstruction method. Another parameter that we have used is the Hubble distance, $D_{H}(z)=1 / H(z)$ from the Sloan Digital Sky Survey (SDSS) data release 12 (DR12) (Bautista et al. 2017) and the final data release of the SDSS-III (Riess et al. 2018).

In order to measure the BAO scale from the clustering of matter, the definition of fiducial cosmology is required so approximately the distance constraints presented in Table (5) are multiplied by a factor $\left(r_{d} / r_{\mathrm{d} \text {, fid }}\right)$, which is the ratio between the sound horizon to the same quantity computed in the fiducial cosmology. We take this ratio as a free parameter in the statistical analysis for both models. We use 14 uncorrelated data points of the Table 5 in our analysis. In Table (6), we reported the results of our statistical analysis for two models by using BAO data.
Table 6. The best values of the two common parameters obtained by using $\mathrm{BAO}$ and $\mathrm{BBN}$ data.

\begin{tabular}{cccc}
\hline Model & $\Omega_{b 0}$ & $H_{0}$ & $\chi^{2}$ \\
\hline MOG & $0.047_{-0.002}^{+0.001}$ & $66.50_{-0.91}^{+0.52}$ & 17.35 \\
\hline$\Lambda \mathrm{CDM}$ & $0.047_{-0.003}^{+0.002}$ & $68.28_{-1.8}^{+1.8}$ & 13.08 \\
\hline
\end{tabular}

Table 7. The best values of the two common parameters obtained with a combination of the $f \sigma_{8}(z)$ dataset (18 points) and BBN data.

\begin{tabular}{ccccc}
\hline Model & $\Omega_{b 0}$ & $H_{0}$ & $\sigma_{80}$ & $\chi^{2}$ \\
\hline MOG & $0.047_{-0.006}^{+0.005}$ & $68.61_{-1.2}^{+0.8}$ & $0.79_{-0.05}^{+0.06}$ & 11.95 \\
\hline$\Lambda \mathrm{CDM}$ & $0.036_{-0.005}^{+0.007}$ & $78.12_{-4.9}^{+6.4}$ & $0.81_{-0.05}^{+0.05}$ & 11.53 \\
\hline
\end{tabular}

\subsection{Redshift Space Distortions}

One of the very important probes of large scale structure is redshift space distortions that they provide measurements of $f \sigma_{8}(a)$. In galaxy redshift surveys, RSD measures the peculiar velocities of matter. The result is inferring the growth rate of cosmological perturbations. This measurement is done on a wide range of redshifts and scales. This can be obtained by measuring the ratio of the monopole and the quadrupole multipoles of the redshift space power spectrum which depends on $\beta=f / b$. Here $f$ is the growth rate and $b$ is the bias The combination of $f \sigma_{8}(a)$ is independent of the bias factor and the bias dependence in this combination cancels out thus. This combination is a good discriminator of the models (Nesseris et al. 2017; Marra et al. 2019).

In this study, we use the vigorous $f \sigma_{8}(z)$ measurements from the "Gold-2017" compilation given in Nesseris et al. (2017, Table III). In this case we can write:

$\chi_{f s}^{2}(\mathbf{p})=\sum_{i} \frac{\left[f \sigma_{8}\left(z_{i}, \mathbf{p}\right)-f \sigma_{8, i}\right]^{2}}{\sigma_{i}^{2}}$. 
In Table (7), we reported results of the best values of the two common parameters obtained with a combination of the $f \sigma_{8}(z)$ dataset and BBN data.

\subsection{Joint analysis}

In this work, we wish to compare the MOG model against the novel observational data. Specifically, we perform an overall statistical analysis using the background(geometrical) data (SN, BAO, CMB, Big Bang Nucleosynthesis, OHD data) and the growth data. So in the first joint analyses, we considered the background data and performed a likelihood analysis by the MCMC algorithm. We listed the best fit values of parameters using the joint analysis of geometry measurements data for MOG and $\Lambda$ CDM models in Table (8). As can be seen, there is a discrepancy between the best values of the two models for $H_{0}$. It seems MOG theory predicted a smaller value for $H_{0}$ which is in disagreement with the local observations of $H_{0}$ like HOLiCOW ( $H_{0}$ Lenses in COSMOGRAIL's Wellspring) experiment in Wong et al. (2020). They have used a joint analysis of six gravitationally lensed quasars with measured time delays to achieve the highest-precision probe of $H_{0}$ and found $H_{0}=73.3 \pm 1.8$ for a flat $\Lambda$ CDM cosmology. Recently Birrer et al. (2020) based on a new hierarchical Bayesian analyses in which the mass sheet transform is only constrained by stellar kinematics have reported new results. They used mock lenses, which are generated from hydrodynamic simulations and in first step, they applied the inference to the TDCOSMO sample of seven lenses, six of which are from $\mathrm{H} 0 \mathrm{LiCOW}$, and measured $H_{0}=74.5_{-6.1}^{+5.6}$. In the second step, they added spectroscopy and imaging for a set of 33 strong gravitational lenses from the Sloan Lens ACS (SLACS) sample in order to more constrain the deflector mass density profiles and they resolved kinematics to constrain the stellar anisotropy for nine of the 33 SLAC lenses. Assuming that the TDCOSMO and SLACS galaxies are derived from the same parent population they measured $H_{0}=67.4_{-3.2}^{+4.1}$ from the joint hierarchical analysis of the TDCOSMO+SLACS sample so we see that the result of the MOG theory is consistent with their reported results. Of course, this difference in the values reported of Birrer et al. (2020) may be due to that the H0LiCOW could be hiding a correlation with the CMB dipole direction as is disccused in Krishnan et al. (2021). They presented the cordinates of lenses on the celestial sphere from H0LiCOW and declination and their value of $H_{0}$.

In the Figure (5) we show the $1 \sigma$ and $2 \sigma$ confidence levels for the parameters $\Omega_{b 0}, H_{0}$ and $r_{\mathrm{d} \text {,fid }}$ that are in common with the $\Lambda$ CDM model. As we see, the contours for $\Omega_{b 0}$ and $H_{0}$ overlap, and the contour of $\Omega_{b 0}$ for the MOG model has expanded to larger values and inversely $H_{0}$ for $\Lambda$ CDM wides to higher values. Interestingly, there is a significant difference between $r_{\mathrm{d} \text {,fid }}$ contours for the two models.

In the second analysis, we also include perturbation observables, 18 RSD independent data points. We repeated statistical analyses and the observational constraints are summarized in Table (10). Contour of the common parameters of the two models in this case, $\left(\Omega_{b 0}, H_{0}, r_{\mathrm{d} \text {,fid }}, \sigma_{80}\right)$, are shown in the Figure (6). Interestingly, the contours in the MOG case shift towards a higher value of $\Omega_{b 0}$ and smaller values of $H_{0}$. We also see that $\sigma_{80}$ for the MOG model relative to $\Lambda \mathrm{CDM}$ shift towards higher value, It is in agreement with other works as Moffat \& Toth (2013), Shojai et al. (2017), where MOG increases the growth rate of matter perturbations, compared to $\Lambda \mathrm{CDM}$.

Additionally, in Figure (7) we concisely display the constraints on the present value of the Hubble parameter $H_{0}$, considering the observational datasets considered in this work as separately and combination.

\section{MODEL SELECTION}

Model selection is the process of choosing and distinguishing candidate models with various free parameters. To select the most compatible model with the observational data, we need numerical procedures that can obtain the goodness of fit and also determine the best value of free parameters. The least squares method is one of the simplest comparisons which have mostly been applied in cosmology. In general, the $\chi^{2}$ method is very popular and sufficient for comparing different models when they have the same number of degree of freedom(d.o.f) , and the model with a smaller $\chi^{2}$ means it has a better fit with the data. However, for models with a different d.o.f, a model with more parameters tends to lead to the lowest $\chi^{2}$. Under these conditions, the $\chi^{2}$ method is unrighteous for comparing models. So in order to test the quality of fit of the present models, an important quantity that is used for the data fitting process is the reduced $\chi^{2}$ as

$\chi_{\text {red }}^{2}=\frac{\chi_{\text {min }}^{2}}{N-K}$,

where $N$ denotes the total number of data points used in the statistical analysis and $K$ the number of free parameters for the model. As an approximate method, when the variance of the measurement error is known a priori, a $\chi_{\text {red }}^{2} \gg 1$ indicates a poor model fit which the fit has not entirely taken the data. Having the value of $\chi_{\text {red }}^{2}$ around 1 indicates that the extent of the match between observations and estimates is in accord with the error variance. A $\chi_{\text {red }}^{2} \ll 1$ indicates that the model is "over-fitting" the data: either the model is inadequately fitting noise, (or the error variance has been overestimated) (Bevington \& Robinson 2003).

Therefore we conclude from the result of the Table (9) the MOG theory $\left(\chi_{\text {red }}^{2}=1.3\right)$ and $\Lambda$ CDM model $\left(\chi_{\text {red }}^{2}=1.2\right)$ are consistent with the observational background data. But in some studies such as Andrae et al. (2010) was discussed that the traps involved in using $\chi_{\text {red. }}^{2}$. There are two independent problems: (a) the number of degrees of freedom can only be estimated for linear models and inverse for nonlinear models, The number of dof is unknown i.e., it is impossible to calculate the value of reduced $\chi_{\text {red }}^{2}$. (b) The value of $\chi_{\text {red }}^{2}$ itself is subject to noise due to random noise in the data, i.e., its value is uncertain. Since this uncertainty impairs the efficiency of reduced chi-squared for differentiating between models or recognizing the convergence of a minimization procedure so we consider two kinds of criteria for model selection: the Akaike Information Criterion (AIC) and Bayesian Information Criterion (BIC) for comparing two models as:

$$
\begin{aligned}
& \mathrm{AIC}=\chi_{\min }^{2}+2 K+\frac{2 K(K+1)}{N-K-1}, \\
& \mathrm{BIC}=\chi_{\min }^{2}+K \ln N .
\end{aligned}
$$

Since $\Lambda \mathrm{CDM}$ has the best agreement to almost observational data so $\Lambda \mathrm{CDM}$ is the best choice to consider a reference 
Table 8. Marginalized constraints ( $1 \sigma$ uncertainties) of free parameters for two models using the background observables of equation (23).

\begin{tabular}{ccccccc}
\hline Model & $\Omega_{b 0}$ & $\Omega_{m 0}$ & $H_{0}$ & $V_{G 0}$ & $\beta$ & $r_{d, f i d}$ \\
\hline \hline MOG & $0.049_{-0.002}^{+0.002}$ & - & $67.80_{-1.1}^{+1.0}$ & $0.89_{-0.08}^{+0.02}$ & $70.06_{-0.002}^{+0.002}$ & $182.4_{-2.1}^{+2.9}$ \\
\hline$\Lambda \mathrm{CDM}$ & $0.044_{-0.001}^{+0.001}$ & $0.26_{-0.02}^{+0.01}$ & $71.10_{-1.0}^{+1.0}$ & - & - & $149.3_{-2.5}^{+2.5}$ \\
\hline
\end{tabular}

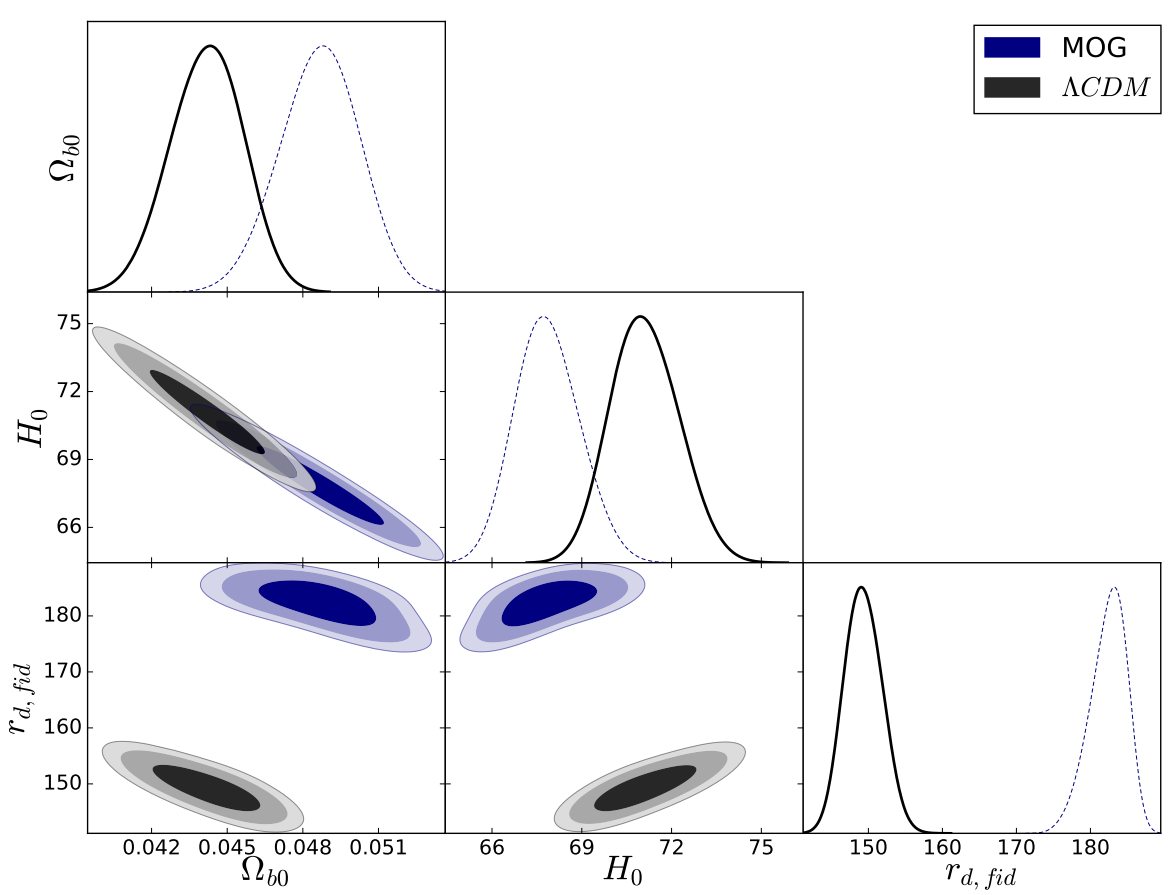

Figure 5. 2D contours and $1 \mathrm{D}$ posterior distributions of the free parameters of MOG model(blue contour) that are in common with the $\Lambda$ CDM model(black contour) relative to background data. using background data(see equation (23)). See Table (8) for the numerical values.

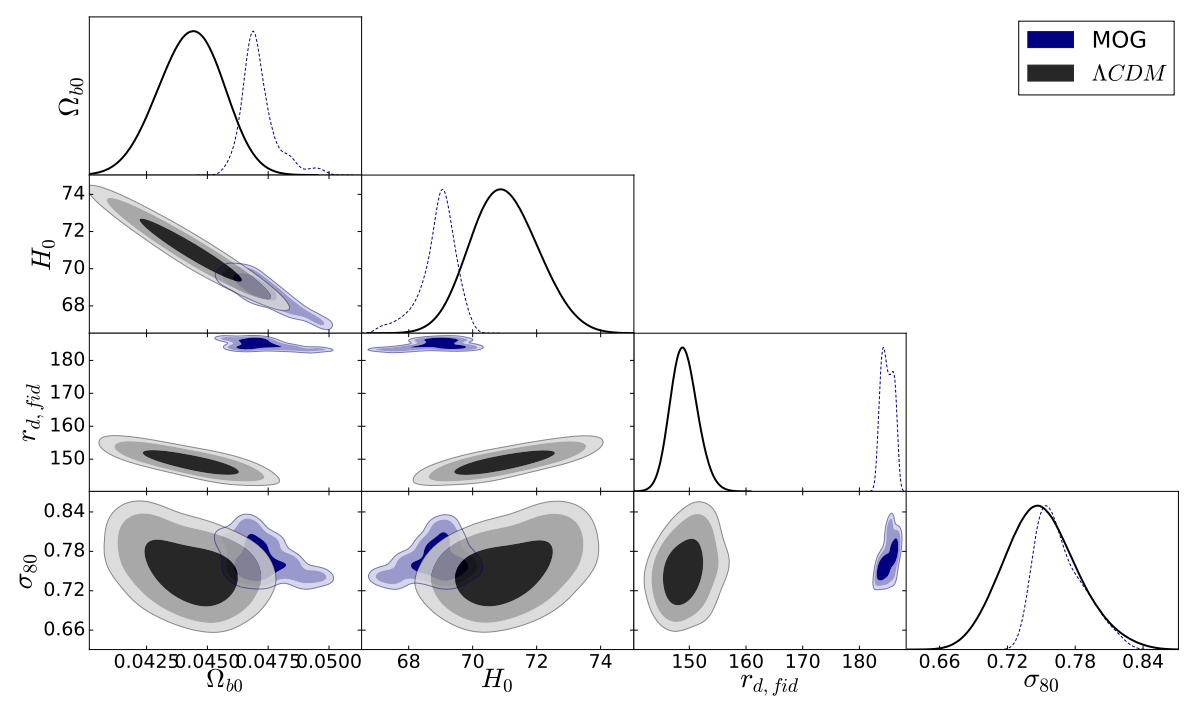

Figure 6. $2 \mathrm{D}$ contours and $1 \mathrm{D}$ posterior distributions of the free parameters for the MOG model(top left panel) and $\Lambda$ CDM model using both background and perturbation data. 
Table 9. Model selection of the statistical results by using the background observables of the equation (23).

\begin{tabular}{ccccccc}
\hline Model & $\chi_{\text {min }}^{2}$ & $\chi_{\text {red }}^{2}$ & $\Delta \chi_{\text {min }}^{2}$ & $\Delta$ AIC & $\Delta$ BIC & $\Delta$ DIC \\
\hline \hline MOG & 110.1 & 1.33 & 3.7 & 5.9 & 8.2 & 3.4 \\
\hline$\Lambda$ CDM & 106.4 & 1.27 & - & - & - & \\
\hline
\end{tabular}

model. Now, for any model $M$, other than the reference model (denoted by $\mathrm{R}$ ), from the difference $\Delta \mathrm{AIC}=\left|\mathrm{AIC}_{\mathrm{M}}-\mathrm{AIC}_{\mathrm{R}}\right|$, we get at the following conclusions of (i) If $\Delta \mathrm{AIC} \leq 2$, then the concerned model has substantial support with respect to the reference model (i.e. it has evidence to be a good cosmological model), (ii) $4 \leq \Delta \mathrm{AIC} \leq 7$ indicates less support with respect to the reference model, and finally, (iii) $\Delta \mathrm{AIC} \geq 10$ means that the model has no support, in fact, it has no use in principle. Similarly, for the BIC a difference in the range $0<\Delta \mathrm{BIC} \leq 2$ is considered as weak evidence, for $2<\Delta \mathrm{BIC} \leq 6$ is considered as positive evidence, and $6<\Delta \mathrm{BIC} \leq 10$ is strong evidence while more than 10 is very strong evidence against the model with the higher BIC.

Another additional information criterion that we describe in the following is the Deviance Information Criterion (DIC). Spiegelhalter (2002) developed this method of model selection in an effort to generalize the AIC. The DIC has some interesting properties. First, it could use for the situation where one or more parameters or a combination of parameters are insignificantly constrained by the data such as in astrophysics, unlike the AIC and BIC. Secondly, it is easily measured from posterior samples, such as those generated by MCMC methods. It has already been used to study quasar clustering in astrophysics, and it is stated as (Liddle 2007; Rezaei \& Malekjani 2021):

$D I C=2 \overline{\chi_{\text {tot }}^{2}(\mathbf{p})}-\chi_{\text {tot }}^{2}(\overline{\mathbf{p}})$.

Therefore in the first analysis that we use the background observational data, the results of Table (9) show that the MOG model has less support compared to the reference model, $\Lambda \mathrm{CDM}$ with observational background data $(\Delta \mathrm{AIC})$ and that there is positive evidence against it $(\Delta \mathrm{BIC})$. Then the evidence in favor of the MOG model becomes a category weaker and indicating strong support for the $\Lambda \mathrm{CDM}$ model. We can conclude from $\Delta$ DIC that both models are equally supported by the data.

In the second analysis, For the background and the growth rate data, our results in Table (11) especially $\Delta$ DIC show that MOG and $\Lambda$ CDM models fit the cosmological data equally well.

\section{COSMOLOGICAL EVOLUTION}

In this section, we illustrate the evolution of basic cosmological values based on the best appropriate values of the cosmological parameters presented in Table (10).

We plot the evolution of $\mathrm{H}(\mathrm{z})$ and $H(z) /(1+z)$ in Figure (8) for best fit parameters in Table (10).

In Figure (9), we present the evolution of the fractional energy densities for different components. We see for two models, the universe evolves from a radiation dominated phase to a matter dominated epoch and the density parameter of

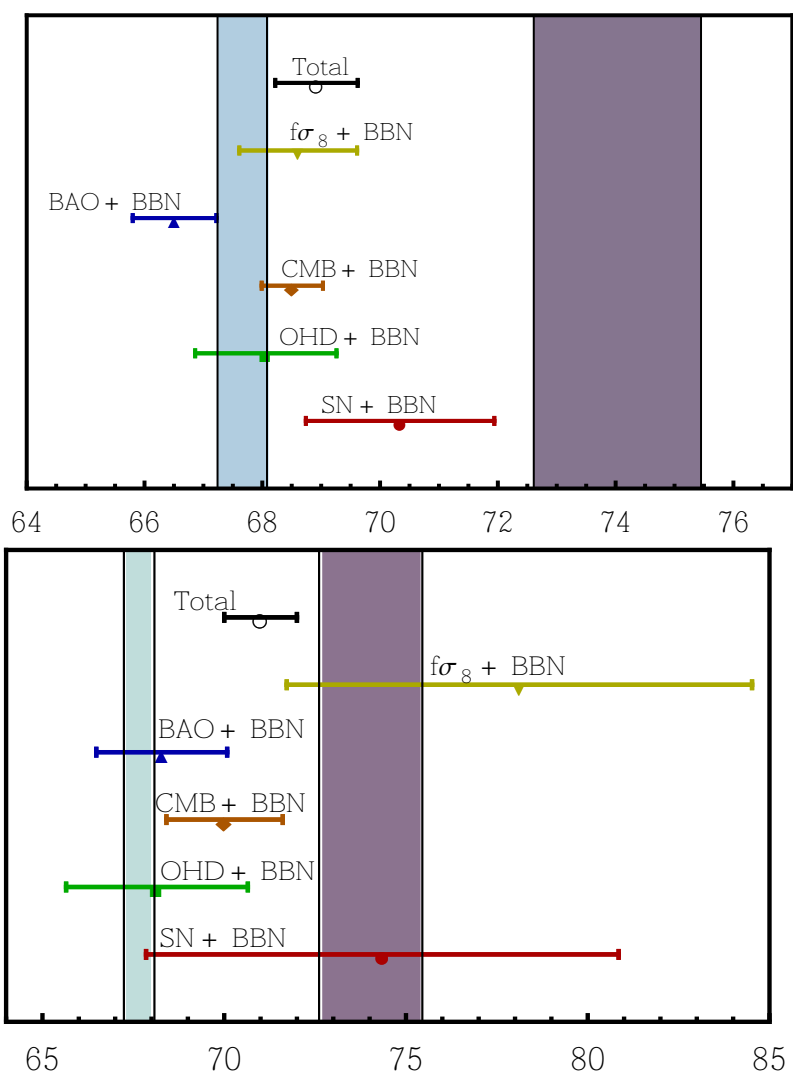

Figure 7. Whisker graph with the $68 \%$ confidence level region for $H_{0}$ for the observational datasets considered in this work as separately and combination for MOG (top panel) and $\Lambda \mathrm{CDM}$ (bottom panel) models. Schematic representation of $H_{0}$ at $1 \sigma$ ( $\mathrm{SN}$ (red color), $\mathrm{OHD}$ (green color), $\mathrm{CMB}$ (orange color), $\mathrm{BAO}$ (blue color), $f \sigma_{8}$ (yellow color) and Total: $\mathrm{SN}+\mathrm{OHD}+\mathrm{BAO}+\mathrm{CMB}+\mathrm{BBN}+f \sigma_{8}$ (black color) ) for MOG (top panel) and $\Lambda$ CDM (bottom panel) models. We also show Planck 2018, $H_{0}=67.66 \pm 0.42 \mathrm{~km} \mathrm{~s}^{-1} \mathrm{Mpc}^{-1}$ (cyan region) by Aghanim et al. (2020) and $H_{0}=74.03 \pm 1.42 \mathrm{~km} \mathrm{~s}^{-1} \mathrm{Mpc}^{-1}$ (gray region) by Riess et al. (2019).

the pressure-less matter decreases and dark energy for $\Lambda \mathrm{CDM}$ model and $V_{G}$ in the MOG model increases by decreasing redshift, indicating the early time matter dominated universe. In Figure (10), we compare the observed $f \sigma_{8}(z)$ with the theoretical value of the growth rate function for two models by the best free parameters. As we expected from statistical analysis in section (4.6) and the results of Table (11), the predicted growth rate in the MOG model can fit the observed $f \sigma_{8}(z)$ as well as $\Lambda$ CDM model. It is consistent with the results of Moffat \& Toth (2013) where they modeled perturbation growth in MOG theory and compared the observed matter power spectrum with the prediction at the present time.

Another parameter we calculated by the best value is the effective EoS and we obtained $w_{\text {eff }, \Lambda}=-0.70$ and $w_{\text {eff }, \mathrm{MOG}}=$ -0.67 and in the Figure (11), we show the evolution in term of redshift. To this end, using the best-fitting values of Table (10), we can calculate the age of the universe via the following expression

$t=\int_{0}^{1} \frac{d a}{a H(a)}$. 
Table 10. Marginalized constraints ( $1 \sigma$ uncertainties) for free parameters of two models using both the background and perturbation data.

\begin{tabular}{cccccccc}
\hline Model & $\Omega_{b 0}$ & $\Omega_{m 0}$ & $H_{0}$ & $V_{G 0}$ & $\beta$ & $r_{d, f i d}$ & $\sigma_{80}$ \\
\hline \hline MOG & $0.047_{-0.001}^{+0.001}$ & - & $68.92_{-0.4}^{+0.7}$ & $0.80_{-0.01}^{+0.02}$ & $70.06_{-0.001}^{+0.001}$ & $185.0_{-1.1}^{+1.1}$ & $0.77_{-0.03}^{+0.02}$ \\
\hline$\Lambda \mathrm{CDM}$ & $0.044_{-0.001}^{+0.001}$ & $0.26_{-0.01}^{+0.01}$ & $71.0_{-1.0}^{+1.0}$ & - & - & $149.2_{-2.5}^{+2.2}$ & $0.75_{-0.04}^{+0.03}$ \\
\hline
\end{tabular}

Table 11. Model selection of the statistical results by using the background and perturbation observables.

\begin{tabular}{ccccccc}
\hline Model & $\chi_{\text {min }}^{2}$ & $\chi_{\text {red }}^{2}$ & $\Delta \chi_{\text {min }}^{2}$ & $\Delta$ AIC & $\Delta$ BIC & $\Delta$ DIC \\
\hline \hline MOG & 122.1 & 1.22 & 2.3 & 4.5 & 6.9 & 0.07 \\
\hline$\Lambda \mathrm{CDM}$ & 119.8 & 1.19 & - & - & - & \\
\hline
\end{tabular}
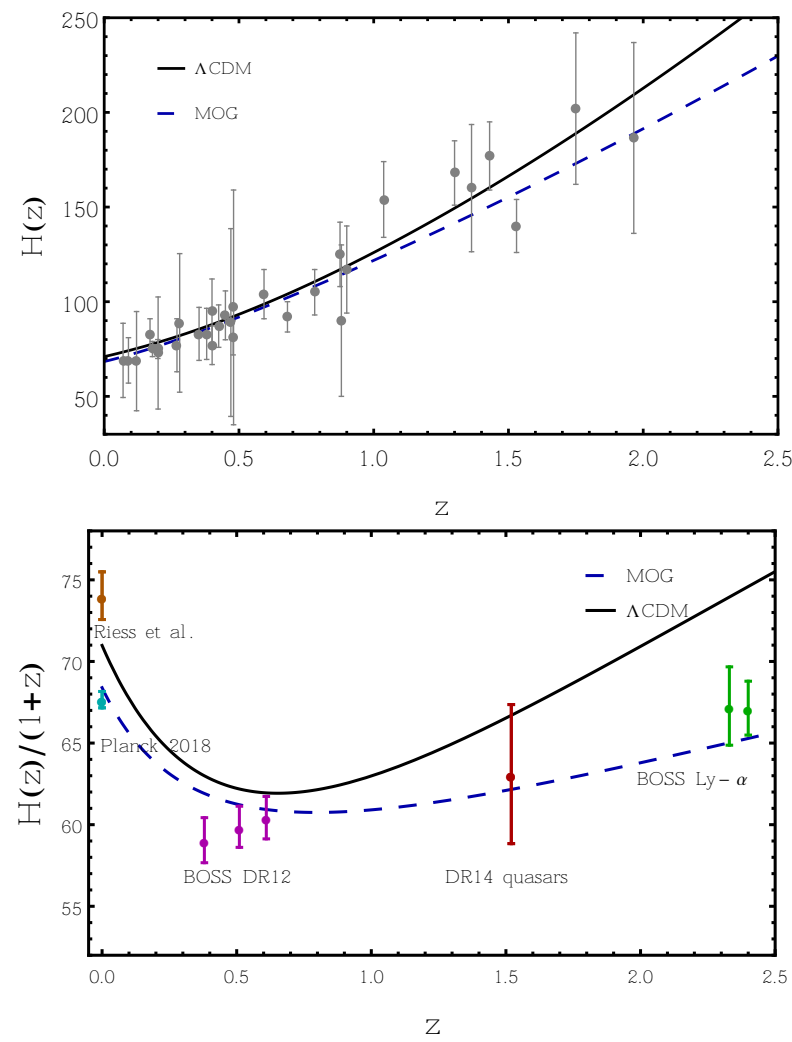

Figure 8. In the top panel, we compared the 31 cosmic chronometer data points in gray dots (Marra \& Sapone 2018) and theoretical redshift evolution of Hubble parameter $\mathrm{H}(\mathrm{z})$. In the bottom panel, we have plotted $\mathrm{H}(\mathrm{z})$ vs. redshift. The black solid curve shows the concordance $\Lambda \mathrm{CDM}$ model.

One of the important quantities in cosmology studies is the age of the universe because it connects $H_{0}$, which can be measured in the late and the early universe. For $\Lambda$ CDM, we find $t_{\Lambda}=13.6$ Gyr which are in a good compatibility with the Planck results: 13.78 Gyr;(Aghanim et al. 2020) and for MOG model we obtain a larger value of $t_{\mathrm{MOG}}=14.4 \mathrm{Gyr}$. In addition to the above equation, the age can also be measured using very old objects. Since the mid-1990s, estimates of the
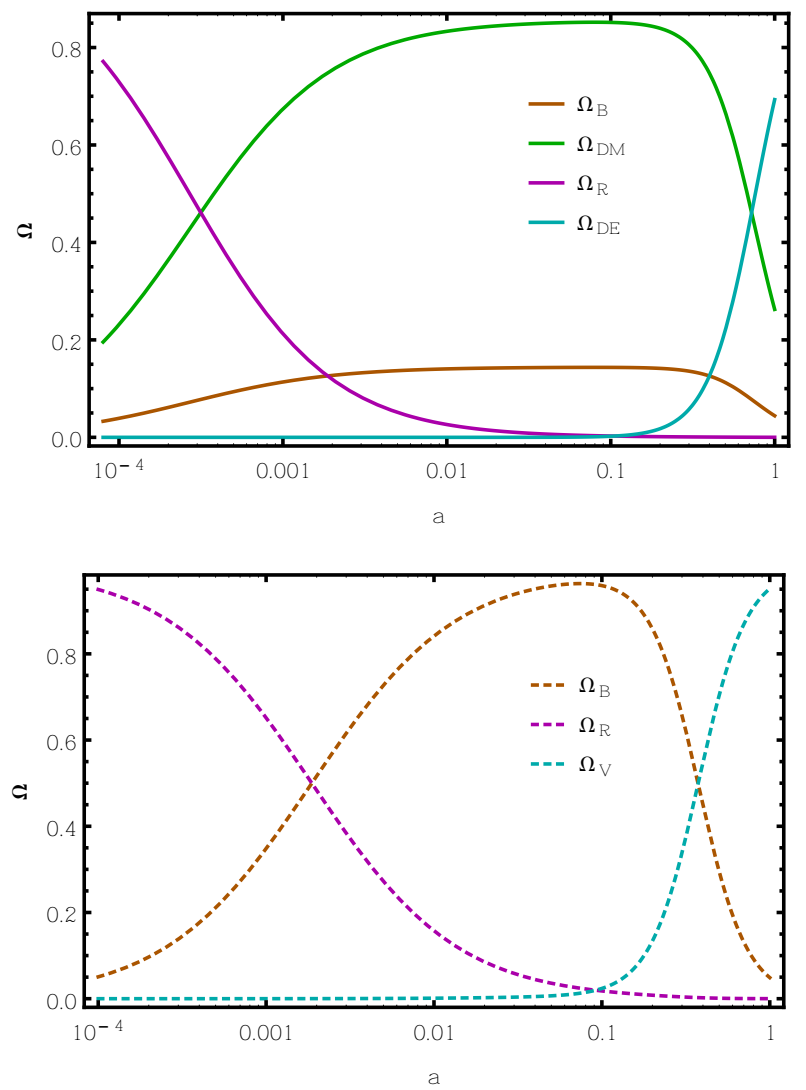

Figure 9. Evolution of the fractional energy density of radiation (violet curves), baryonic matter (orange curves), and DE component (blue curves) in terms of a cosmic scale factor for standard $\Lambda \mathrm{CDM}$ (top panel) and MOG(bottom panel) cosmologies. The solid green curve represents dark matter component of the concordance $\Lambda \mathrm{CDM}$ model. In both models, we use the best fit values from Table (10).

ages of globular clusters have consistently been in the range 12-14 Gyr(Bailin et al. 1996). For example, recently it is obtained $t=13.35 \pm 0.56$ Gyr using populations of stars in globular clusters(Valcin et al. 2020). Despite the robust and rigorous testing that has been done extensively for CMB, BAO, and SN, we need to be more careful about the age of the oldest objects. For example, the age of the oldest stars 2MASS $\mathrm{J} 18082002-5104378 \mathrm{~B}$ is measured as $t_{\star}=13.535 \pm 0.002 \mathrm{Gyr}$, but if the scatter among different models to fit for the age is taken into account the age becomes $t_{\star}=13.0 \pm 0.6 \mathrm{Gyr}$, and for HD 140283 is equaled to $t_{\star}=14.46 \pm 0.8 \mathrm{Gyr}$, but becomes $t_{\star}=13.5 \pm 0.7$ Gyr using the new Gaia parallaxes instead of original HST parallaxes (Di Valentino et al. 2020). The last panel in this study is shown in Figure (12) where 


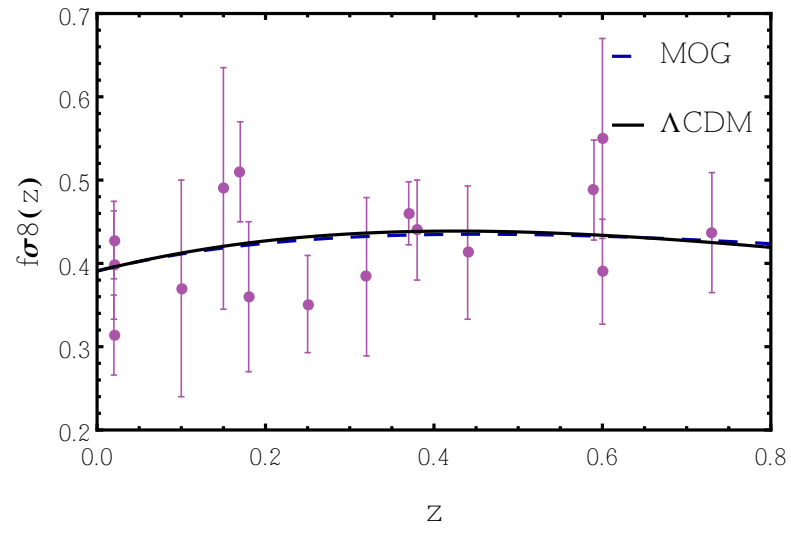

Figure 10. The evolution of $f \sigma_{8}(z)$ as a function of the cosmic redshift and the "Gold-2017" growth rate dataset. The blue dashed and the black solid curves correspond to the best fits of MOG and $\Lambda$ CDM models, respectively. See Table (10) for the numerical value of free parameters.

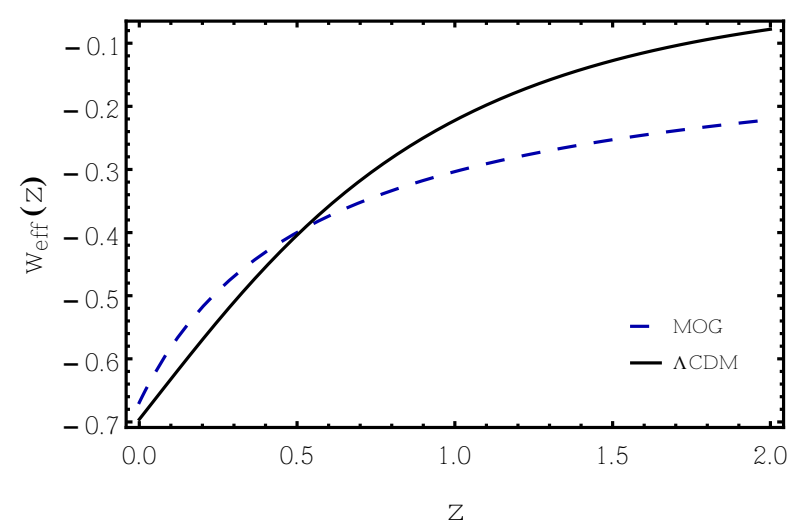

Figure 11. The evolution of $w_{\text {eff }}(z)$ as a function of the cosmic redshift. See Table (10) for the numerical value of free parameters.

we compared the 114 observational data points reported in Vagnozzi et al. (2021) for the ages of old astrophysical objects (OAO) in range $z<8$ with theoretical redshift evolution of the age of the universe for both MOG theory and the concordance $\Lambda \mathrm{CDM}$ model using their best free parameters that were reported in Table (10). As we see two models are consistent with these data.

Thus, even if there is no real tension between the different age of the universe determinations at present, most of the error-bars in the age determination comes from the fact that different stellar models do not really agree with each other at the required level of precision to be really able to help with the tensions in cosmology.

\section{CONCLUSIONS}

In this paper, we surveyed the MOG theory as a possible alternative candidate for DE and DM components in cosmology. We performed the present study by a three-step process. First, we solved the system of the basic differential equations (Friedmann and Jeans) at the background and perturbation

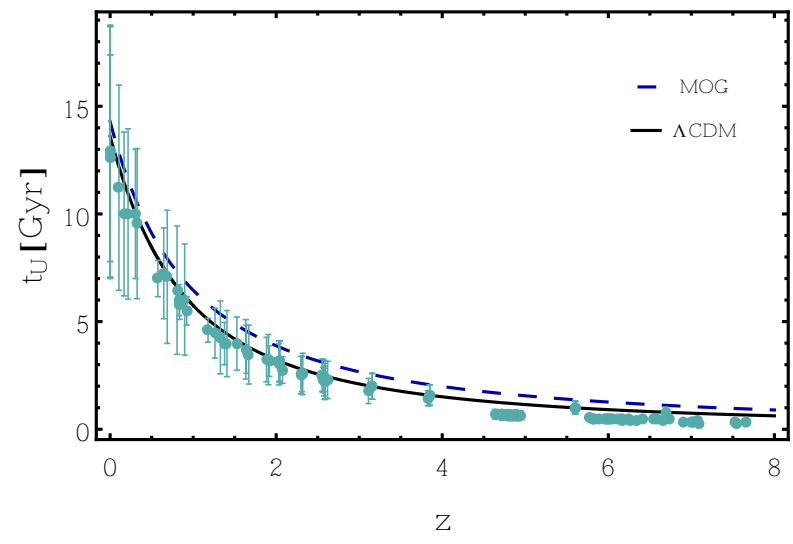

Figure 12. The evolution of the age of the universe, $t_{U}$ for both MOG theory (the blue dashed curve) and the concordance $\Lambda$ CDM (the black solid curve) model as a function of the cosmic redshift using best free parameters was reported in Table (10) and the observational data points were reported in (Vagnozzi et al. 2021) for the ages of old astrophysical objects (OAO).

levels. We investigated the behavior of the basic cosmological quantities $\left(\Omega(z), H(z), q(z), w_{\mathrm{eff}}(z)\right)$ in the background level( see Figures (1) \& (2)) and $\left(f(z), \sigma_{8}(z), f \sigma_{8}(z)\right)$ in the perturbation level (see Figure (3)) in order to understand the global characteristics of the MOG model for different values of its free parameter, $\beta$. As expected, the cosmic expansion depends on the value of $\beta$.

Secondly, we performed a combined statistical analysis, involving the novel geometrical data (BBN, OHD, SNe Type Ia,BAO and CMB shift parameter) and growth data $\left(f \sigma_{8}(z)\right)$ and found that the joint and combined statistical analysis, within the context of flat FLRW space can put sturdy constraints on the basic cosmological parameters. As we reported in Table (10), we see that $\Omega_{b 0}$ and $\sigma_{80}$ for the MOG model relative to $\Lambda$ CDM shift towards a higher value. We found a significant difference between the values of the sound horizon at the baryon drag epoch, $r_{d, f i d}$ between MOG and $\Lambda \mathrm{CDM}$ models.

Thirdly, using the Deviance Information Criterion and the reduced $\chi^{2}$, we concluded that both MOG and $\Lambda$ CDM models fit the observational data well.

Lastly, using the aforementioned best cosmological parameters, we found that the age of the universe is greater in the MOG model than in the $\Lambda \mathrm{CDM}$ model. Within the context of background dynamics of the universe and growth of structure in the linear regime, MOG is compatible with the observed data.

\section{ACKNOWLEDGMENTS}

This work has been supported financially by Iran Science Elites Federation under research project No. M/99101. The authors gratefully thank John Moffat and Savvas Nesseris for valuable discussion. 


\section{DATA AVAILABILITY}

No new data were generated or analysed in support of this research.

\section{References}

Abbott T. M. C., et al., 2019, Mon. Not. Roy. Astron. Soc., 483, 4866

Aghanim N., et al., 2018

Aghanim N., et al., 2020, Astron. Astrophys., 641, A6

Alam S., et al., 2020, arXiv:2007.08991

Andrae R., Schulze-Hartung T., Melchior P., 2010

Ata M., et al., 2018, Mon. Not. Roy. Astron. Soc., 473, 4773

Bailin D., Love A., Sabra W. A., Thomas S., 1996, Phys. Lett. B, 378, 113

Bautista J. E., et al., 2017, Astron. Astrophys., 603, A12

Bernui A., Novaes C. P., Pereira T. S., Starkman G. D., 2018

Betoule M., et al., 2014, Astron. Astrophys., 568, A22

Bevington P. R., Robinson D. K., 2003, Data reduction and error analysis for the physical sciences. (2d ed.; New York: McGraw Hill)

Birrer S., et al., 2020, Astron. Astrophys., 643, A165

Brownstein J. R., Moffat J. W., 2006, Astrophys. J., 636, 721

Camarena D., Marra V., 2018, Phys. Rev., D98, 023537

Carolina N., et al., 2020, JCAP, 07, 015

Chen L., Huang Q.-G., Wang K., 2019, JCAP, 02, 028

Davari Z., Rahvar S., 2020, Mon. Not. Roy. Astron. Soc., 496, 3502

Davari Z., Marra V., Malekjani M., 2020, Mon. Not. Roy. Astron. Soc., 491, 1920

Di Valentino E., et al., 2020, arXiv:2008.11286

Farooq O., Madiyar F. R., Crandall S., Ratra B., 2017, Astrophys. J., 835,26

Green M. A., Moffat J. W., 2019, Phys. Dark Univ., 25, 100323

Green M. A., Moffat J. W., Toth V. T., 2018, Phys. Lett. B, 780, 300

Hinshaw G., et al., 2013, Astrophys. J. Suppl., 208, 19

Hrycyna O., Szydłowski M., Kamionka M., 2014, Phys. Rev. D, 90, 124040

Hu W., Sugiyama N., 1996, ApJ, 471, 542

Israel N. S., Moffat J. W., 2018, Galaxies, 6, 41

Jamali S., Roshan M., Amendola L., 2020, Phys. Lett. B, 802, 135238

Jimenez R., Loeb A., 2002, Astrophys. J., 573, 37

Kazin E. A., et al., 2014, Mon. Not. Roy. Astron. Soc., 441, 3524

Krishnan C., Mohayaee R., Colgáin E. O., Sheikh-Jabbari M. M., Yin L., 2021

Kumar S., Nunes R. C., Yadav S. K., 2019, Eur. Phys. J. C, 79, 576

Liddle A. R., 2007, Mon. Not. Roy. Astron. Soc., 377, L74

Marra V., Sapone D., 2018, Phys. Rev., D97, 083510

Marra V., Rosenfeld R., Sturani R., 2019, Universe, 5

McDonald P., et al., 2005, Astrophys. J., 635, 761

Moffat J. W., 2006, JCAP, 0603, 004

Moffat J. W., 2014, arXiv:1409.0853

Moffat J. W., 2020, Eur. Phys. J. C, 80, 906

Moffat J. W., Rahvar S., 2013, MNRAS, 436, 1439

Moffat J. W., Rahvar S., 2014, MNRAS, 441, 3724

Moffat J. W., Toth V. T., 2007, arXiv:0710.0364

Moffat J., Toth V., 2009, Class. Quant. Grav., 26, 085002

Moffat J. W., Toth V. T., 2013, Galaxies, 1, 65

Moffat J., Toth V., 2015, Phys. Rev. D, 91, 043004

Moresco M., Jimenez R., Verde L., Pozzetti L., Cimatti A., Citro A., 2018, Astrophys. J., 868, 84

Nesseris S., Pantazis G., Perivolaropoulos L., 2017, Phys. Rev., D96, 023542

Nunes R. C., Bernui A., 2020, Eur. Phys. J. C, 80, 1025
Pérez J. d. C., 2021, Other thesis (arXiv:2105.14800)

Rata B., Peebles J., 1988, Phys. Rev. D, 37, 321

Rezaei M., Malekjani M., 2021, Eur. Phys. J. Plus, 136, 219

Riess A. G., et al., 2018, Astrophys. J., 855, 136

Riess A. G., Casertano S., Yuan W., Macri L. M., Scolnic D., 2019, Astrophys. J., 876, 85

Ross A. J., Samushia L., Howlett C., Percival W. J., Burden A., Manera M., 2015, Mon. Not. Roy. Astron. Soc., 449, 835

Schwarz D. J., Copi C. J., Huterer D., Starkman G. D., 2016, Class. Quant. Grav., 33, 184001

Scolnic D., et al., 2018, Astrophys. J., 859, 101

Serra P., Cooray A., Holz D. E., Melchiorri A., Pandolfi S., et al., 2009, Phys. Rev. D, 80, 121302

Shojai F., Cheraghchi S., Bouzari Nezhad H., 2017, Physics Letters B, 770,43

Slosar A., et al., 2013, JCAP, 1304, 026

Solà Peracaula J., Gómez-Valent A., de Cruz Pérez J., MorenoPulido C., 2020, Class. Quant. Grav., 37, 245003

Spiegelhalter David J. e. a., 2002, J. R.Statist. Soc. B., 64, 583

Suzuki N., et al., 2012, Astrophys. J., 746, 85

Toth V. T., 2010, in International Conference on Two Cosmological Models. (arXiv:1011.5174)

Vagnozzi S., Pacucci F., Loeb A., 2021

Valcin D., Bernal J. L., Jimenez R., Verde L., Wandelt B. D., 2020, JCAP, 12, 002

Wong K. C., et al., 2020, Mon. Not. Roy. Astron. Soc., 498, 1420 